Delft University of Technology

\title{
On the relevance of kinematics for cavitation implosion loads
}

Schenke, S.; Melissaris, T.; Van Terwisga, T. J.C.

DOI

10.1063/1.5092711

Publication date

2019

Document Version

Final published version

Published in

Physics of Fluids

\section{Citation (APA)}

Schenke, S., Melissaris, T., \& Van Terwisga, T. J. C. (2019). On the relevance of kinematics for cavitation implosion loads. Physics of Fluids, 31(5), [052102]. https://doi.org/10.1063/1.5092711

\section{Important note}

To cite this publication, please use the final published version (if applicable).

Please check the document version above.

\section{Copyright}

Other than for strictly personal use, it is not permitted to download, forward or distribute the text or part of it, without the consent of the author(s) and/or copyright holder(s), unless the work is under an open content license such as Creative Commons.

\section{Takedown policy}

Please contact us and provide details if you believe this document breaches copyrights.

We will remove access to the work immediately and investigate your claim. 


\section{On the relevance of kinematics for cavitation implosion loads}

Cite as: Phys. Fluids 31, 052102 (2019); https://doi.org/10.1063/1.5092711

Submitted: 13 February 2019 . Accepted: 11 April 2019. Published Online: 03 May 2019

S. Schenke (D), T. Melissaris (D), and T. J. C. van Terwisga (iD)

\section{ARTICLES YOU MAY BE INTERESTED IN}

Cavitation and ventilation modalities during ditching

Physics of Fluids 31, 052101 (2019); https://doi.org/10.1063/1.5092559

A numerical study on bubble dynamics in sinusoidal channels

Physics of Fluids 31, 052103 (2019); https://doi.org/10.1063/1.5092870

Geometric optimization of riblet-textured surfaces for drag reduction in laminar boundary layer flows

Physics of Fluids 31, 053601 (2019); https://doi.org/10.1063/1.5090881

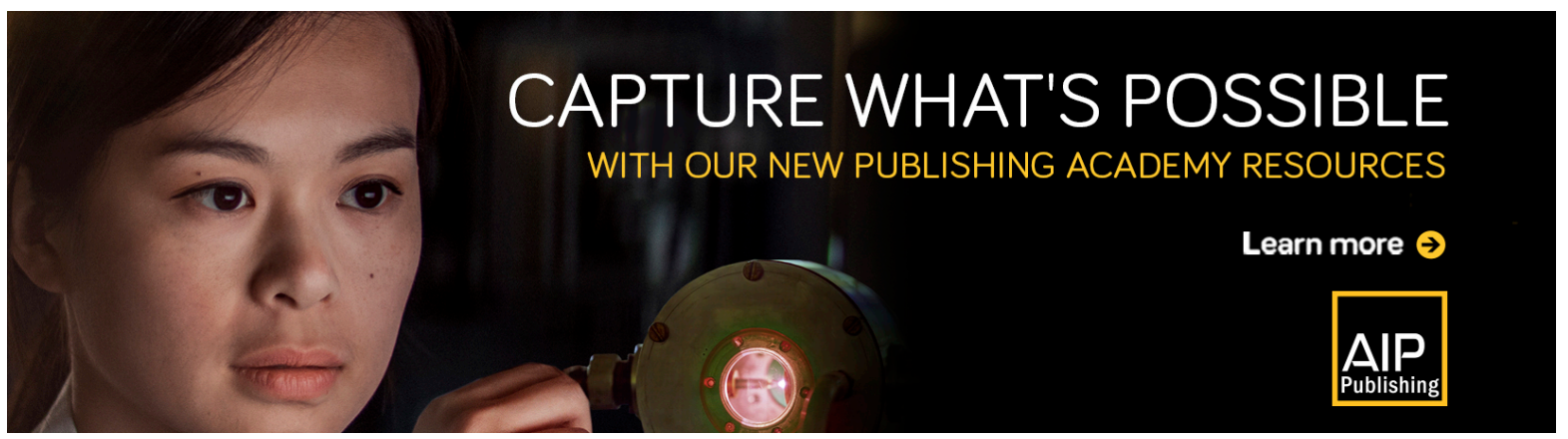




\title{
On the relevance of kinematics for cavitation implosion loads
}

\author{
Cite as: Phys. Fluids 31, 052102 (2019); doi: 10.1063/1.509271 1 \\ Submitted: 13 February 2019 • Accepted: 11 April 2019 • \\ Published Online: 3 May 2019
}

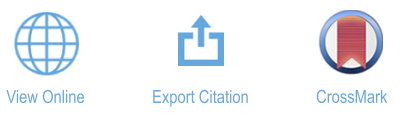

S. Schenke, ${ }^{1, a)}$ (D) T. Melissaris, ${ }^{1,2}$ (D) and T. J. C. van Terwisga ${ }^{1,3}$ (iD)

\begin{abstract}
AFFILIATIONS
${ }^{1}$ Maritime and Transport Technology Department, Delft University of Technology, 2628 CD Delft, The Netherlands

${ }^{2}$ Department of Hydrodynamics, Wärtsilä Netherlands B.V., 5151 DM Drunen, The Netherlands

${ }^{3}$ R\&D Team "Resistance \& Propulsion," Maritime Research Institute Netherlands (MARIN), 6708 PM Wageningen,

The Netherlands
\end{abstract} a) Also at: Process and Energy Department, Delft University of Technology, Delft, The Netherlands. Electronic mail: s.schenke@
tudelft.nl.

\begin{abstract}
This study presents a novel physical model to convert the potential energy contained in vaporous cavitation into local surface impact power and an acoustic pressure signature caused by the violent collapse of these cavities in a liquid. The model builds on an analytical representation of the solid angle projection approach by Leclercq et al. ["Numerical cavitation intensity on a hydrofoil for 3D homogeneous unsteady viscous flows," Int. J. Fluid Mach. Syst. 10, 254-263 (2017)]. It is applied as a runtime post-processing tool in numerical simulations of cavitating flows. In the present study, the model is inspected in light of the time accurate energy balance during the cavity collapse. Analytical considerations show that the potential cavity energy is first converted into kinetic energy in the surrounding liquid [D. Obreschkow et al., "Cavitation bubble dynamics inside liquid drops in microgravity," Phys. Rev. Lett. 97, 094502 (2006)] and focused in space before the conversion into shock wave energy takes place. To this end, the physical model is complemented by an energy conservative transport function that can focus the potential cavity energy into the collapse center before it is converted into acoustic power. The formulation of the energy focusing equation is based on a Eulerian representation of the flow. The improved model is shown to provide physical results for the acoustic wall pressure obtained from the numerical simulation of a close-wall vapor bubble cloud collapse.

(C) 2019 Author(s). All article content, except where otherwise noted, is licensed under a Creative Commons Attribution (CC BY) license (http://creativecommons.org/licenses/by/4.0/). https://doi.org/10.1063/1.5092711
\end{abstract}

\section{NOMENCLATURE}

$\mathbf{u}$ flow velocity vector $(\mathrm{m} / \mathrm{s})$

$\mathbf{x}_{P}, \mathbf{x}_{S} \quad$ inner volume/surface location vector $(\mathrm{m})$

n surface normal vector (-)

$C_{c}, C_{v} \quad$ condensation/evaporation constant $\left(\mathrm{kg} \mathrm{s} / \mathrm{m}^{5}\right)$

$c_{l} \quad$ liquid sound speed $(\mathrm{m} / \mathrm{s})$

$d, \partial, D$ general, partial, and material derivative operator

E energy (J)

$e \quad$ energy per volume $\left(\mathrm{J} / \mathrm{m}^{3}\right)$

$e_{S} \quad$ energy per surface area $\left(\mathrm{J} / \mathrm{m}^{2}\right)$

$\mathcal{E} \quad$ collapse induced kinetic energy per volume $\left(\mathrm{J} / \mathrm{m}^{3}\right)$

$h \quad$ energy focusing parameter (-)

p absolute/total pressure $(\mathrm{Pa})$ $p_{d}, p_{a} \quad$ driving pressure, acoustic pressure $(\mathrm{Pa})$

$p_{\infty}, p_{v}$ ambient pressure, vapor pressure $(\mathrm{Pa})$

$\mathcal{P} \quad$ power $(\mathrm{W})$

$\mathfrak{P}_{\mathbf{b}}(\mathbf{a}) \quad$ normalized projection of vector $\mathbf{a}$ on vector $\mathbf{b}$ such that $\mathfrak{P}_{\mathbf{b}}(\mathbf{a})=\mathbf{a} \cdot \mathbf{b} /(\|\mathbf{a}\|\|\mathbf{b}\|)$

$R, r \quad$ bubble radius, distance from bubble center $(\mathrm{m})$

$S \quad$ surface area $\left(\mathrm{m}^{2}\right)$

$t, \tau, T$ time, collapse time, wave period (s)

$\delta t, \Delta t \quad$ time increment, simulation time step size (s)

$V \quad$ volume $\left(\mathrm{m}^{3}\right)$

$\beta \quad$ radiated energy fraction (-)

$\delta \quad$ small number added to a denominator to prevent from division by 0

$\theta \quad$ angle ( $\mathrm{rad})$ 
$\rho, \rho_{l}, \rho_{v} \quad$ density of mixture, liquid, vapor $\left(\mathrm{kg} / \mathrm{m}^{3}\right)$

$\phi \quad$ spatial transport operator/function

$\Omega \quad$ solid angle (sr)

\section{Subscripts/superscripts}

$\begin{array}{ll}\text { pot } & \text { potential } \\ \text { rad } & \text { radiated } \\ \text { kin } & \text { kinetic } \\ S W & \text { shock wave } \\ b & \text { bubble } \\ C & \text { cell center } \\ f & \text { cell face } \\ 0 & \text { initial }\end{array}$

\section{INTRODUCTION}

Cavitation is a multiphase flow phenomenon that is often encountered in fluid machinery such as marine propulsion systems, water turbines, or gear pumps. It typically occurs at locations of high flow velocities, where the pressure may drop to such low values that the liquid evaporates. The violent collapse of cavitating structures in regions of pressure recovery causes potentially erosive surface impact loads. The assessment of cavitation erosion risk from numerical flow simulation is still a major challenge because the erosive aggressiveness of such impact loads strongly depends on flow phenomena acting on extremely small scales in space and time. In the present study, a novel methodology is introduced, which is specifically designed to predict the cavitating flow aggressiveness from the dynamics of the larger scale cavitating structures. The approach builds up on previous work ${ }^{1,2}$ and is motivated by the potential energy hypothesis, ${ }^{3,4}$ which essentially states that the initial potential energy of an imploding cavity is proportional to the pressure difference driving the cavity collapse and the initial cavity volume. While numerous approaches ${ }^{5-9}$ have been developed based on the potential energy hypothesis, practically none of them have specifically addressed the question as to how the conversion of potential energy into local surface impact power can be achieved such that the overall energy balance is satisfied.

A step toward incorporating the energy balance of the cavity collapse into a cavitation erosion model applicable to numerical flow simulations has been achieved by Leclercq et al., ${ }^{1}$ who have developed a cavitation intensity model to compute the local impact power caused by imploding cavities. In its essence, the radiated power computed from the local change of specific cavity volume is projected on the solid surface in this approach. A fully continuous form of this model has previously been developed, ${ }^{10}$ which has allowed to derive analytical predictions on the amount of accumulated surface energy, against which the numerical results can be verified. ${ }^{10}$ Even though it has been shown that the overall energy balance holds at the end of a cavity collapse, the cavitation intensity approach still misses out to reflect the spatial and temporal focusing of the potential cavity energy, as described by Tinguely et al. ${ }^{11}$

Based on the works by Wang and Brennen, ${ }^{12}$ Obreschkow et al., ${ }^{13}$ and Tinguely et al., ${ }^{11}$ it will be discussed how the cascade of energy during the collapse can approximately be reduced to the focusing of the initial potential cavity energy into the collapse center and the subsequent conversion into acoustic power. The cavitation intensity model is complemented by an energy focusing equation that can achieve this spatial focusing of potential cavity energy. The model is applied to a cloud of 125 vapor bubbles collapsing under a high ambient pressure of 40 bars. The numerical test case had been set up earlier by Schmidt et al. ${ }^{14}$ where they investigated the characteristic of a pressure signal on a numerical pressure sensor located on a solid surface underneath the collapsing cloud. The results by Schmidt et al. ${ }^{14}$ which they have kindly shared together with the bubble cloud specifications, are considered a reliable reference data set because it has been obtained from a fully compressible densitybased numerical approach and because grid size independence of the results has been demonstrated for a fixed acoustic Courant number. ${ }^{14}$ To test the complemented cavitation intensity model against the results by Schmidt et al., ${ }^{14}$ an acoustic analogy is employed which allows us to reconstruct the acoustic pressure from the cavitation intensity approach. The model is implemented as a runtime post-processing tool in the open source CFD (computational fluid dynamics) environment OpenFOAM. ${ }^{1}$

\section{CAVITATION INTENSITY}

\section{A. Cavitating flow modeling}

Following up on the study by Schmidt et al. ${ }^{14}$ viscous and surface tension forces are assumed to be negligible for the highly inertia driven flow problem considered in this study. The Euler equations for momentum and mass continuity to be solved are then given by

$$
\begin{gathered}
\frac{\partial}{\partial t}(\rho \mathbf{u})+\nabla \cdot(\rho \mathbf{u} \otimes \mathbf{u})=-\nabla p, \\
\frac{\partial \rho}{\partial t}+\nabla \cdot(\rho \mathbf{u})=0 .
\end{gathered}
$$

With $\gamma$ being the liquid fraction, the density of the cavitating flow is given by the linear mixture relation

$$
\rho=\gamma \rho_{l}+(1-\gamma) \rho_{v}, \quad \text { where } 0 \leq \gamma \leq 1 .
$$

The pure liquid and vapor phases are modeled as incompressible. In the mixture regime, the velocity divergence is modeled by a source term which is given by a slightly modified version ${ }^{16}$ of the cavitation model by Merkle et al. ${ }^{17}$ Combining the mass continuity equation given by Eq. (2) with the mixture relation given by Eq. (3) and the modified Merkle model gives the transport equation of $\gamma$ that must be solved to achieve phase transition ${ }^{16}$

$$
\frac{\partial \gamma}{\partial t}+\nabla \cdot(\gamma \mathbf{u})=\frac{p-p_{v}}{\rho \rho_{l}}\left\{\begin{array}{cl}
C_{v} \gamma, & \text { if } p \leq p_{v} \\
C_{c}(1-\gamma), & \text { if } p>p_{v} .
\end{array}\right.
$$

\section{B. Acoustic power and pressure}

Let the radiated volume specific power radiated at some location $\mathbf{x}_{P}$ and some time instant $t$ be $\dot{e}_{\text {rad }}\left(t, \mathbf{x}_{P}\right)$. The instantaneous surface specific impact power at some surface location $\mathbf{x}_{S}$ resulting from the distribution of all emission sources in the domain is then given by ${ }^{10}$

$$
\dot{e}_{S}\left(t, \mathbf{x}_{S}\right)=\frac{1}{4 \pi} \int_{\mathrm{vol}} \dot{e}_{\mathrm{rad}}\left(t, \mathbf{x}_{P}\right)\left[\frac{\left(\mathbf{x}_{P}-\mathbf{x}_{S}\right) \cdot \mathbf{n}}{\left\|\mathbf{x}_{P}-\mathbf{x}_{S}\right\|^{3}}\right] d V .
$$

As illustrated in Fig. 1, $\mathbf{n}$ is the surface normal vector at the impact location. Equation (5) is a fully continuous form of the solid angle 


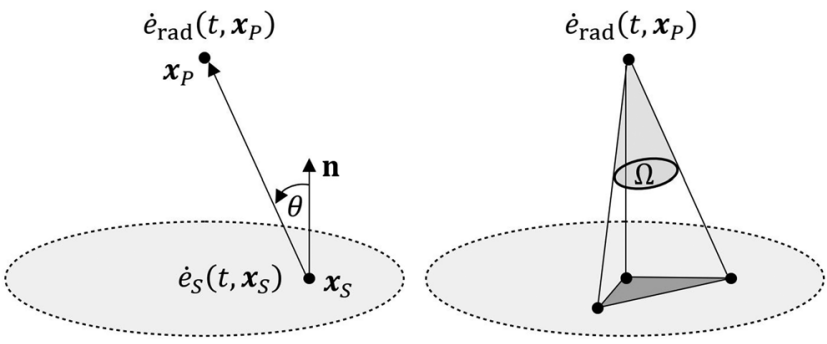

FIG. 1. Coordinate definitions for the conversion of the volume specific power radiated at location $\mathbf{x}_{p}$ into local surface specific impact power at the surface location $\mathbf{x}_{S}$ (left) and illustration of the solid angle projection of radiated power on triangular surface elements as proposed by Leclercq et al. ${ }^{1}$ (right).

projection approach by Leclercq et al. ${ }^{1}$ Fully continuous means that Eq. (5) represents the surface specific impact power at a point location, whereas Leclercq et al. ${ }^{1}$ employ the solid angle $\Omega$ (see Fig. 1) to project the radiated power on triangular surface elements of finite size. Nevertheless, both formulations represent an energy conservative conversion of radiated energy into local surface impact power. As Eq. (5) represents the surface specific impact power associated with a pressure wave, it can be rewritten in terms of the acoustic power density $d \mathcal{P} / d S$ such that

$$
\dot{e}_{S}\left(t, \mathbf{x}_{S}\right)=\frac{d \mathcal{P}}{d S}=\frac{p_{a}^{2}}{\rho_{l} c_{l}} \cos \theta .
$$

In Eq. (6), $\rho_{l}$ and $c_{l}$ are the liquid density and sound speed, respectively, and $p_{a}$ is the acoustic pressure perturbation. The term $\cos \theta$ reflects the orientation of the reference surface $d S$ relative to the propagation direction of the encountered sound wave. In Eq. (5), $\cos \theta$ is represented by the relation $\cos \theta=\left(\mathbf{x}_{P}-\mathbf{x}_{S}\right) \cdot \mathbf{n} /\left|\mathbf{x}_{P}-\mathbf{x}_{S}\right|$. Combining Eqs. (5) and (6) with the relation for $\cos \theta$ allows to reconstruct the acoustic pressure perturbation from the acoustic analogy

$$
p_{a}\left(t, \mathbf{x}_{S}\right)=\frac{1}{2} \sqrt{\frac{\rho_{l} c_{l}}{\pi} \int_{\text {vol }} \frac{\dot{e}_{\text {rad }}\left(t, \mathbf{x}_{P}\right)}{\left\|\mathbf{x}_{P}-\mathbf{x}_{S}\right\|^{2}} d V .}
$$

For a source impacting a flat surface stretched to infinity (see Fig. 1), it has been shown analytically that the surface and time integrated impact power, hence the accumulated surface energy, becomes

$$
E_{S}(\tau)=\frac{1}{2} E_{\mathrm{pot}, 0}
$$

as the collapse time $\tau$ is approached. Consequently, half of the initial potential cavity energy is eventually impacting the flat surface. ${ }^{2}$

\section{Energy balance during the cavity collapse}

According to the potential energy hypothesis, ${ }^{3,4}$ the potential energy of a cavity is equal to the work that the driving pressure difference $p_{d}-p_{v}$ can do on its vapor volume throughout the collapse, where $p_{d}$ is the driving pressure and $p_{v}$ is the vapor pressure. During the collapse, the initial potential cavity energy $E_{\text {pot }, 0}$ is partitioned into different forms of energy, however, such that the total energy amount $E_{\text {pot, } 0}$ is conserved at any time instant. ${ }^{11,13}$ In order to discuss the relevance of the instantaneous energy balance with respect to the cavitation intensity approach, we will first employ the simplifying case of an undisturbed vapor bubble collapse. In this case, the driving pressure $p_{d}$ is equal to the ambient pressure $p_{\infty}$ and the potential cavity energy reduces to the potential bubble energy ${ }^{4}$

$$
E_{\text {pot }, 0}^{b}=\frac{4}{3} \pi R_{0}^{3}\left(p_{\infty}-p_{v}\right)
$$

where $R_{0}$ is the initial bubble radius and where the superscript $b$ indicates that the relation is valid for a spherical undisturbed bubble. In order to formulate the instantaneous energy balance during the collapse process, the entire liquid body around the collapsing bubble needs to be taken into account. ${ }^{13}$ As the bubble collapses, its potential energy decreases. The reduction of potential energy feeds into kinetic energy of the liquid body. The local volume specific kinetic energy at some distance $r$ from the center is $1 / 2 \rho_{l}|\mathbf{u}|^{2}(r, t)$. Due to the spherical symmetry of the problem, it is convenient to integrate the volume specific kinetic energy over the surface of a sphere around the bubble center, which gives the radial kinetic energy density. With the mass continuity relation $r^{2}|\mathbf{u}|=R^{2} \dot{R}$, the instantaneous radial kinetic energy density at some distance $r$ from the bubble center becomes

$$
\frac{d E_{\mathrm{kin}}}{d r}=2 \pi \rho_{l} R^{4} \frac{\dot{R}^{2}}{r^{2}} \quad \text { for } \quad r \geq R
$$

where $R=R(t)$ is the instantaneous bubble radius. The instantaneous kinetic energy of the entire liquid body follows from the radial integration of Eq. (10), which gives

$$
E_{\text {kin }}^{b}=\int_{R}^{\infty}\left(\frac{d E_{\text {kin }}}{d r}\right) d r=2 \pi \rho_{l} \dot{R}^{2} R^{3}
$$

and, hence, the instantaneous change of kinetic energy around the collapsing bubble ${ }^{18}$

$$
\frac{d E_{\mathrm{kin}}^{b}}{d t}=2 \pi \rho_{l} R^{2} \dot{R}\left[2 R \ddot{R}+3 \dot{R}^{2}\right] .
$$

From Eq. (9) follows the change of potential energy

$$
\frac{d E_{\mathrm{pot}}^{b}}{d t}=4 \pi R^{2} \dot{R}\left(p_{\infty}-p_{v}\right) .
$$

As pointed out by Obreschkow et al., ${ }^{13}$ the change of kinetic energy in the liquid body and the change of potential bubble energy must cancel each other out such that $d E_{\text {pot }}^{b} / d t+d E_{\text {kin }}^{b} / d t=0$, which gives the Rayleigh equation ${ }^{13,19}$

$$
R \ddot{R}+\frac{3}{2} \dot{R}^{2}=-\frac{p_{\infty}-p_{v}}{\rho_{l}} .
$$

Substituting the analytical solution ${ }^{19}$

$$
\dot{R}=-\sqrt{\frac{2}{3} \frac{p_{\infty}-p_{v}}{\rho_{l}}\left(\frac{R_{0}^{3}}{R^{3}}-1\right)}
$$

into Eq. (11), one finds that

$$
E_{\text {kin }}^{b}(R=0)=E_{\text {pot }, 0}^{b} .
$$

This means that the initial potential bubble energy is fully converted into kinetic energy as the final collapse stage is approached. Also, 
the kinetic energy around a spherical collapsing bubble focuses toward the bubble interface over time. This is shown by combining Eq. (10) with the analytical solution for $\dot{R}$ given by Eq. (15), which gives the dimensionless radial kinetic energy density at some distance $r$ from the collapse center

$$
\left(\frac{d E_{\mathrm{kin}}^{b}}{d r}\right)^{*}=\frac{R_{0}}{E_{\mathrm{pot}, 0}} \frac{d E_{\mathrm{kin}}^{b}}{d r}=\left(\frac{R_{0}}{r}\right)^{2} \frac{R}{R_{0}}\left[1-\left(\frac{R}{R_{0}}\right)^{3}\right] .
$$

Evaluating Eq. (17) at the interface shows that $\left.\lim _{R \rightarrow 0}\left(d E_{\text {kin }}^{b} / d r\right)^{*}\right|_{r=R}$ $=\infty$, which means that the radial kinetic energy density at the bubble interface tends toward infinity as the final collapse stage is approached. As we further know from Eq. (16) that the kinetic energy of the entire liquid body around the bubble approaches the finite value $E_{\mathrm{pot}, 0}^{b}$, we can conclude that all the kinetic energy of the liquid body focuses into the collapse center at the final collapse stage. The focusing of kinetic energy is illustrated by Fig. 2, which depicts the radial distribution of the dimensionless kinetic energy density given by Eq. (17) for different bubble radii, hence for different time instants of the bubble collapse. According to these simplifying considerations, the shock wave emitted at the final collapse stage propagates from an idealized point source. The relation between the power radiation $\dot{e}_{\text {rad }}$ associated with the shock and the acoustic pressure $p_{a}$ at some distance $r=\left|\mathbf{x}_{P}-\mathbf{x}_{S}\right|$ from the source is obtained by rewriting Eq. (7) for an isolated point source, which gives

$$
\left.p_{a}^{2}(t, r)\right|_{\mathbf{x}_{P}}=\frac{\rho_{l} c_{l}}{4 \pi r^{2}} \dot{e}_{\mathrm{rad}}(t)
$$

With $T_{S W}$ being the pulse duration at some distance $r$ from the source, the radiated shock wave energy is then given by ${ }^{20}$

$$
E_{S W}=\frac{4 \pi r^{2}}{\rho_{l} c_{l}} \int_{T_{S W}} p_{a}^{2}(t, r) d t .
$$

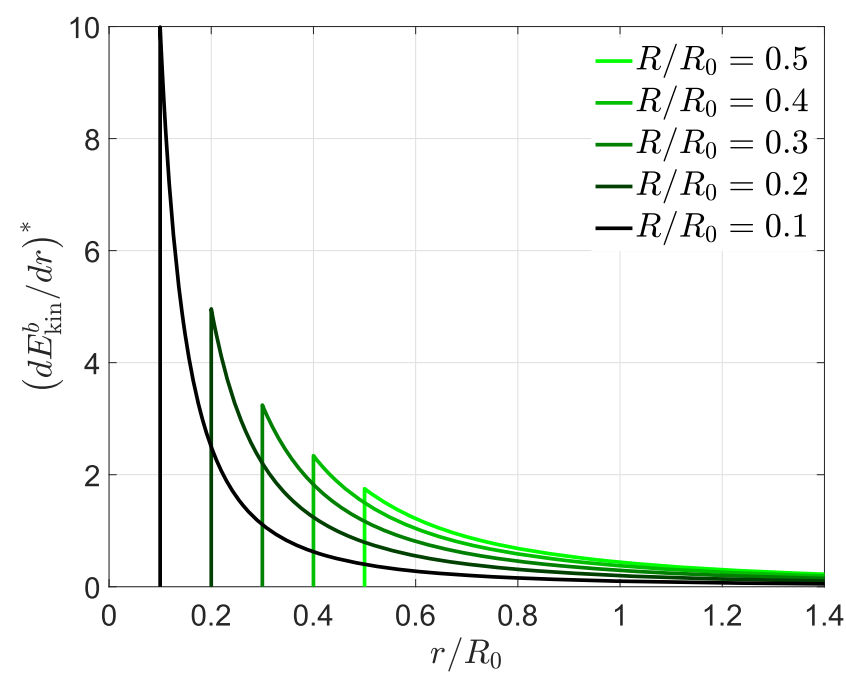

FIG. 2. Distribution of the dimensionless radial kinetic energy density around a collapsing vapor bubble [see Eq. (17)] for different time instants associated with the corresponding bubble radii $R / R_{0}$; the integral of each curve represents the kinetic energy of the entire liquid body, as given by Eq. (11).
As discussed by Tinguely et al., ${ }^{11}$ the initial potential bubble energy is eventually partitioned into shock wave energy, dissipative thermal energy, and rebound energy, where they show the latter to be relevant for low ambient pressures significantly below 1 bar only. Thermal dissipation has been shown to be negligible. ${ }^{11}$ Under these circumstances, the shock wave energy $E_{S W}$ is approximately equal to the initial potential bubble energy $E_{\text {pot }, 0}^{b}$. In addition to the energy balance by Tinguely et al. ${ }^{11}$ which addresses the collapse of spherical bubbles in particular, we also mention that some residual kinetic energy may remain in the flow due to asymmetries in the collapse. This residual kinetic energy may again transform into potentially erosive energy forms, e.g., the waterhammer and stagnation pressure imposed by the impinging liquid jet forming during the collapse of a close wall vapor bubble. ${ }^{21}$ It is further important to mention that the presence of noncondensable gas affects the collapse energy partition. ${ }^{11}$ However, the effect of noncondensable gas has been neglected in the reference case study by Schmidt et al. ${ }^{14}$ and is also not the subject of the present study.

\section{Approaching the time accurate energy balance}

In previous work, ${ }^{1,2}$ it has been assumed that the potential cavity energy is continuously converted into radiated energy throughout the cavity collapse, whereas it has been shown in Sec. II $C$ that the potential energy is gradually converted into kinetic energy ${ }^{13}$ and focused into the collapse center, where it is eventually released in a shock wave of higher amplitude, accordingly. The practical implication is that the events at the final collapse stage can be reflected by the cavitation intensity approach more realistically if only the potential energy itself is somehow accumulated into the collapse center before being released. Against this background, an approach is introduced that allows the focusing of the potential energy into the collapse center and which can also reflect the dynamics of a collective cloud collapse, as illustrated by Fig. 3. In a collective cloud collapse as described by Wang and Brennen, ${ }^{12}$ the potential energy

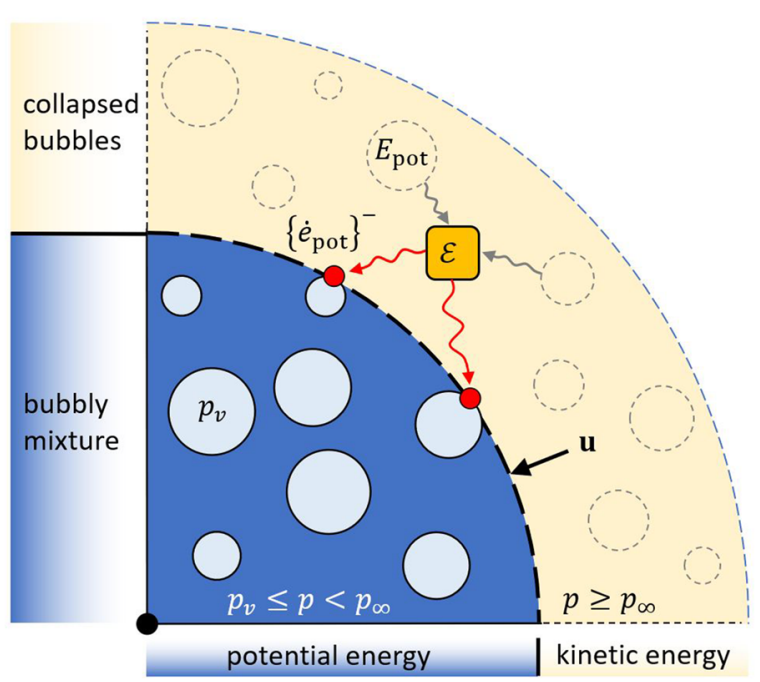

FIG. 3. Illustration of the energy cascade governing the focusing of potential energy. 
of the entire collective vapor bubble cloud is focused into the cloud collapse center.

The reduction of potential cavity energy due to condensation is absorbed in an accumulated kinetic energy field $\mathcal{E}\left(t, \mathbf{x}_{P}\right)$ until a criterion for the conversion of this kinetic energy into radiated acoustic energy is met. This process can formally be expressed by the continuity equation

$$
\frac{\partial \mathcal{E}}{\partial t}+\mathbf{u} \cdot \nabla \mathcal{E}=\underbrace{-\mathcal{E}\{\nabla \cdot \mathbf{u}\}^{-}}_{\text {kin. energy source }}-\underbrace{\dot{e}_{\text {rad }}}_{\text {radiation source }},
$$

where the product rule $\nabla \cdot(\mathcal{E} \mathbf{u})=\mathbf{u} \cdot \nabla \mathcal{E}+\mathcal{E} \nabla \cdot \mathbf{u}$ has been applied. The kinetic energy represented by $\mathcal{E}$ is induced by the volume reduction of the vapor cavities in the flow. Consequently, this collapse induced kinetic energy is generated at locations of negative velocity divergence only, denoted by $\{\nabla \cdot \mathbf{u}\}^{-}$. For further reference, the \{\}$^{+}$and \{\}$^{-}$operator applied to some scalar quantity $\varphi$ is defined as

$$
\{\varphi\}^{+} \equiv \max [\varphi, 0], \quad\{\varphi\}^{-} \equiv \min [\varphi, 0] .
$$

Conversely, a positive velocity divergence associated with cavity growth does not withdraw kinetic energy from the balance, which means that an external energy intake into the system is needed to create the imploding vapor cavities in the first place. Therefore, the two terms on the right-hand side of Eq. (20) can be interpreted as source terms in the kinetic energy balance. They represent the conversion of potential cavity energy into kinetic energy and the conversion of collapse induced kinetic energy into radiated acoustic energy, respectively. Physical models are needed to describe both processes. The term $\mathbf{u} \cdot \nabla \mathcal{E}$ on the left-hand side of Eq. (20) represents the conservative advective transport of $\mathcal{E}$, which is responsible for the spatial focusing of the accumulated kinetic energy. It is known that the amount of collapse induced kinetic energy in the entire liquid body, given by $\int_{\text {vol }} \mathcal{E}\left(t, \mathbf{x}_{P}\right) d V$, is equal to the change of potential energy that the collapsing cavities have undergone. The exact spatial distribution of $\mathcal{E}\left(t, \mathbf{x}_{P}\right)$, however, is unknown. For this reason, the conservative transport of the accumulated kinetic energy requires a modeling assumption as well. We essentially assume that locations of potential energy reduction act as attractors of the collapse induced kinetic energy that is already present in the flow field. Based on this consideration, we further assume that this kinetic energy can be absorbed by the interface of the collapsing cavities until a criterion for the conversion of this energy into radiated acoustic energy is met. Consequently, the model cannot correctly reflect the spatial kinetic energy distribution around the cavity before the final collapse stage. At the final collapse stage, however, where the kinetic energy has been shown to entirely focus into the collapse center, the model approaches the correct representation of the kinetic energy distribution because the cavity interface, in which the kinetic energy is stored, collapses into the center as well. This process can also be thought of as a cascade of kinetic energy, as schematized in Fig. 3.

A model representation of Eq. (20) is given by

$$
\frac{\partial \mathcal{E}}{\partial t}=(1-\beta) \underbrace{\left[\phi(\mathcal{E})-\left.\dot{e}_{\text {pot }}\right|_{\nabla \cdot \mathbf{u} \leq 0}\right]}_{\text {kin. energy flux }}-\underbrace{\beta \frac{\mathcal{E}}{\delta t}}_{\dot{e}_{\text {rad }}} .
$$

The term $\phi(\mathcal{E})$ on the right-hand side of Eq. (22) represents the cascade of the collapse induced kinetic energy and can be seen as a model of the unknown advective transport term $\mathbf{u} \cdot \nabla \mathcal{E}$ in Eq. (20). The term $\left.\dot{e}_{\text {pot }}\right|_{\nabla \cdot \mathbf{u} \leq 0}$ is a model of the kinetic energy source term $\mathcal{E}\{\nabla \cdot \mathbf{u}\}^{-}$in Eq. (20), and it is associated with the local rate of potential energy reduction due to negative flow velocity divergence, which is feeding into kinetic energy. Thus, $\phi(\mathcal{E})-\left.\dot{e}_{\text {pot }}\right|_{\nabla \cdot \mathbf{u} \leq 0}$ represents the kinetic energy flux. The radiated volume specific power $\dot{e}_{\text {rad }}$ is given by a fraction $0 \leq \beta \leq 1$ of the accumulated energy $\mathcal{E}$ radiated within a certain time $\delta t$. The fraction $\beta$ is still to be specified but formally represents a local flow condition that triggers the conversion into radiated acoustic energy. With the radiation source term being proportional to $\beta$, the term $\phi(\mathcal{E})-\left.\dot{e}_{\text {pot }}\right|_{\nabla \cdot \mathbf{u} \leq 0}$ must be multiplied by $(1-\beta)$ to consistently split the kinetic energy flux on the one hand and the radiation energy flux on the other hand in such a way that the overall amount of energy is conserved.

The reduction of potential energy is the result of the driving pressure difference $p_{d}-p_{v}$ acting on the specific vapor volume approximated by $(1-\gamma)$, where $p_{d}$ is the pressure effectively driving the cavity collapse $\mathrm{e}^{22}$

$$
\left.\dot{e}_{\mathrm{pot}}\right|_{\nabla \cdot \mathbf{u} \leq 0}=\underbrace{\{\nabla \cdot \mathbf{u}\}^{-} \frac{\rho}{\rho_{l}-\rho_{v}}}_{-\{D \gamma / D t\}^{+}}\left(p_{d}-p_{v}\right) .
$$

The underbraced term in Eq. (23) is the material derivative of $\gamma$.

In order to derive an appropriate model for the conservative transport term $\phi(\mathcal{E})$ in Eq. $(22), \phi(\mathcal{E})$ is formally decomposed into a positive and a negative contribution such that

$$
\phi(\mathcal{E})=\{\phi(\mathcal{E})\}^{+}+\{\phi(\mathcal{E})\}^{-} .
$$

For the amount of transported energy to be conserved, the transport term must satisfy

$$
\int_{\mathrm{vol}}\left[\{\phi(\mathcal{E})\}^{+}+\{\phi(\mathcal{E})\}^{-}\right] d V=0 .
$$

It is first assumed that the reduction rate given by $\{\phi(\mathcal{E})\}^{-}$is proportional to $\mathcal{E}$ to ensure that $\mathcal{E} \geq 0$ everywhere. Second, the fraction of $\mathcal{E}$ reduced by $\{\phi(\mathcal{E})\}^{-}$per time $\delta t$ is assumed to be given by the normalized projection of $\nabla \mathcal{E}\left(\mathbf{x}_{P}\right)$ on the local flow velocity vector $\mathbf{u}\left(\mathbf{x}_{P}\right)$, which gives

$$
\begin{gathered}
\{\phi(\mathcal{E})\}^{-}=-\frac{\mathcal{E}}{\delta t} \underbrace{\frac{\{\mathbf{u} \cdot \nabla \mathcal{E}\}^{+}}{\|\mathbf{u}\|\|\nabla \mathcal{E}\|}}_{\mathfrak{P}_{\mathbf{u}}(\nabla \mathcal{E})} \\
\text { at any location } \mathbf{x}_{P} \text { where }\|\mathbf{u}\|>0 \wedge\|\nabla \mathcal{E}\|>0 \\
\text { and anywhere else }\{\phi(\mathcal{E})\}^{-}=0, \\
\text { such that } 0 \leq \mathfrak{P}_{\mathbf{u}}(\nabla \mathcal{E}) \leq 1 .
\end{gathered}
$$

For further reference, the underbraced term in Eq. (26) formally denotes the normalized projection of $\nabla \mathcal{E}$ on $\mathbf{u}$. This formulation is motivated by the assumption that the flow at the interface of a collapsing cavity is directed into the collapse center and, hence, aligned with $\nabla \mathcal{E}$ because $\mathcal{E}$ is stored in the cavity interface. The amount of accumulated energy that is reduced by $\{\phi(\mathcal{E})\}^{-}$must be absorbed by the $\{\phi(\mathcal{E})\}^{+}$term. The underlying assumption for the formulation of the $\{\phi(\mathcal{E})\}^{+}$term is that the kinetic energy accumulated throughout the cavity collapse is attracted by locations of negative velocity divergence, where $\left.\dot{e}_{\text {pot }}\right|_{\nabla \cdot \mathbf{u} \leq 0}<0$. To this end, 
it is assumed that $\{\phi(\mathcal{E})\}^{+}$is proportional to $\left.\dot{e}_{\text {pot }}\right|_{\nabla \cdot \mathbf{u} \leq 0}$ such that $\{\phi(\mathcal{E})\}^{+}=\left.k \dot{e}_{\text {pot }}\right|_{\nabla \cdot \mathbf{u} \leq 0}$, where $k$ is assumed to be constant in space and must be determined such that

$$
\left.k \int_{\text {vol }} \dot{e}_{\text {pot }}\right|_{\nabla \cdot \mathbf{u} \leq 0} d V=-\int_{\text {vol }}\{\phi(\mathcal{E})\}^{-} d V
$$

at any time instant to comply with the conservation requirement given by Eq. (25). Thus, we get

$$
\begin{aligned}
&\{\phi(\mathcal{E})\}^{+}=\left.\dot{e}_{\mathrm{pot}}\right|_{\nabla \cdot \mathbf{u} \leq 0} \underbrace{\frac{\int_{\mathrm{vol}} \frac{\mathcal{E}}{\delta t} \mathfrak{P}_{\mathbf{u}}(\nabla \mathcal{E}) d V}{\left.\int_{\mathrm{vol}} \dot{e}_{\mathrm{pot}}\right|_{\nabla \cdot \mathbf{u} \leq 0} d V}}_{k} \\
& \text { at any location } \mathbf{x}_{P} \text { where }\left.\int_{\text {vol }} \dot{e}_{\text {pot }}\right|_{\nabla \cdot \mathbf{u} \leq 0} d V>0 \\
& \text { and anywhere else }\{\phi(\mathcal{E})\}^{+}=0 .
\end{aligned}
$$

A conceptually similar mechanism to describe the interaction between cavitation bubbles based on the volume distribution of the velocity divergence field has been proposed by Maiga et al. ${ }^{2}$ Combining the above equations, Eq. (22) becomes

$$
\frac{\partial \mathcal{E}}{\partial t}=(1-\beta) \underbrace{\left[\left.\dot{e}_{\text {pot }}\right|_{\nabla \cdot \mathbf{u} \leq 0}(k-1)-\frac{\mathcal{E}}{\delta t} \mathfrak{P}_{\mathbf{u}}(\nabla \mathcal{E})\right]}_{\text {kin. energy flux }}-\underbrace{\beta \frac{\mathcal{E}}{\delta t}}_{\dot{e}_{\text {rad }}},
$$

where $\left.\dot{e}_{\text {pot }}\right|_{\nabla \cdot \mathbf{u} \leq 0}, \mathfrak{P}_{\mathbf{u}}(\nabla \mathcal{E})$ and $k$ are specified by Eqs. (23), (26), and (28), respectively.

Finally, it is assumed that the fraction $\beta$ is related to the local flow conditions and given by

$$
\beta=\left(\gamma \min \left[\frac{p}{p_{\infty}}, 1\right]\right)^{h}, \quad \text { where } \quad 0 \leq \beta \leq 1 .
$$

According to Eq. (30), the fraction $\beta$ depends on the local pressure magnitude relative to the ambient pressure $p_{\infty}$ and the local liquid fraction. The liquid fraction criterion is motivated by the thermodynamic states of a water-vapor mixture, which imply that pressure must be close to vapor pressure in the phase transition regime and that a high amplitude pressure wave can only form in the liquid phase. The $p / p_{\infty}$ term reflects the circumstance that the cascade of collapse induced kinetic energy may also take place across the liquid phase in a collective bubble cloud collapse, as illustrated in Fig. 3. In such a situation, the collective cloud collapse is driven by an inward directed bubbly shock as described by Wang and Brennen, ${ }^{12}$ where the pressure on the downstream side of the shock front is supposed to be smaller than $p_{\infty}$. The exponent $h$ in Eq. (30) is the energy focusing parameter. In the lower limit $h \rightarrow 0$, for which $\beta=1$ for any $\gamma>0 \wedge p>0$, all the volume specific energy at the corresponding location is released instantaneously. In the limit $h \rightarrow \infty$, the radiation of energy is suppressed until the local specific volume is fully occupied by liquid and the local pressure exceeds the ambient pressure $p_{\infty}$. The potential energy is then most efficiently focused toward the collapse center, where it is eventually radiated in a rather sudden step. For $h \rightarrow \infty$, Eq. (30) can be rewritten as follows:

$$
\beta=\left\{\begin{array}{ll}
1, & \text { if } \\
0, & \text { else. }
\end{array} p>p_{\infty} \text { and } \gamma=1,\right.
$$

The energy radiation criterion given by Eq. (31) in combination with the potential energy focusing mechanism identifies the final collapse stage in a similar way as proposed in the collapse detector method by Mihatsch et al. ${ }^{24}$ If the flow conditions determining the value of $\beta$ allow for it, the initial potential cavity energy can be entirely focused into the collapse center. From Eq. (29) then follows that $\dot{e}_{\text {rad }} \rightarrow \infty$ as $\delta t \rightarrow 0$. This is the equivalent situation to the events at the final collapse stage of an isolated vapor bubble as found from the simplifying considerations in Sec. II C, where the energy density in the collapse center tends to infinity at the final collapse stage, however, with the absolute amount of energy being finite. This behavior is the result of not resolving the events at the finite collapse stage, which eventually determine the exact energy density distribution within the radiated pressure wave. This energy density distribution is formally reflected by Eq. (19), where the total amount of shock wave energy $E_{S W}$ is proportional to the time integral of $p_{a}$ over the impact duration $T_{S W}$ as the wave passes some arbitrary location at a distance $r$ from the center. Practically, the time scale $\delta t$ will be equal to the time step size $\Delta t_{\text {model }}$ at which the cavitation intensity model is run in the numerical flow simulation. With the complemented cavitation intensity model, the shock wave energy $E_{S W}$ is consistently predicted, but the exact temporal distribution of $p_{a}$ and the corresponding impact duration $T_{S W}$ is beyond the resolution of the model. Consequently, the potential energy focusing approach is supposed to improve the time accuracy of local acoustic power and acoustic pressure signals in the sense that the impact is more realistically focused toward the final collapse stage while still keeping control over the overall energy balance. However, an entirely time accurate solution for the shape of the peak impact cannot be expected from the method.

\section{NUMERICAL SETUP}

\section{A. Computational grid, inital, and boundary conditions}

The bubble cloud consists of 125 nonintersecting bubbles, as depicted in Fig. 4. The positions and corresponding radii $(0.70 \mathrm{~mm}-$ $1.65 \mathrm{~mm}$ ) are the same as in the study by Schmidt et al., ${ }^{14}$ who have

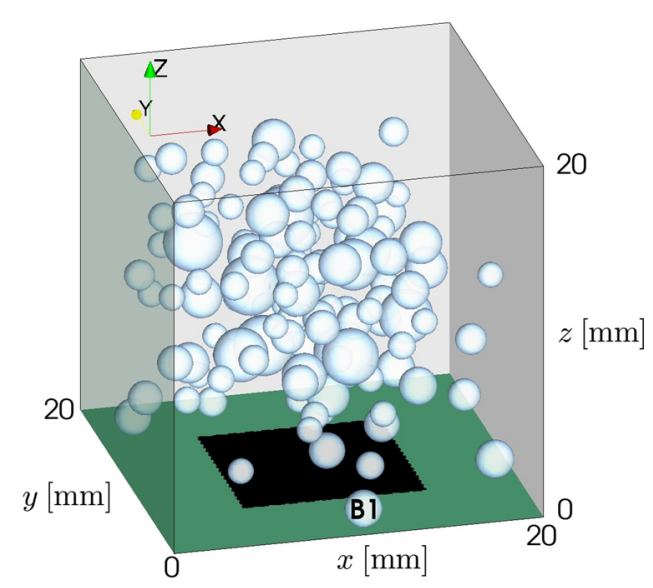

FIG. 4. Bubble cluster and $1 \mathrm{~cm} \times 1 \mathrm{~cm}$ numerical pressure sensor indicated in black on the bottom surface. 
generated a random spatial and bubble size distribution, however, such that the population density increases toward the cloud center. The so obtained data set has kindly been provided by TUM/AER (see Schmidt et al. ${ }^{14}$ ) in private communication as part of the EU H2020 CaFE Project. The cloud is embedded in a cubic inner domain of $20 \mathrm{~mm}$ edge length. The bottom surface of the inner domain is highlighted in green color, and the numerical pressure sensor located right in the middle of the bottom surface is indicated in black color. The pressure sensor signal is obtained by averaging the reconstructed acoustic pressure $p_{a}$ over the sensor surface. The entire computational domain is depicted in Fig. 5. With the bottom surface area and height being $4 \mathrm{~m} \times 4 \mathrm{~m}$ and $2 \mathrm{~m}$, respectively, it is as large that the far field boundaries can be assumed to be undisturbed. The computational mesh is block structured and consists of uniform cubic cells in the inner domain. The outer domain, connecting the inner domain with the far field boundaries, consists of hexahedral cells. The number of cell layers between the inner domain and the far field boundaries is 25 , where the cell expansion ratio toward the far field boundaries is determined such that a smooth cell size transition to the inner domain is achieved. In order to perform a grid sensitivity study, the inner domain (Fig. 6) is systematically refined in $x, y$, and $z$ directions. Three different grid resolutions are investigated in this study. Following the sensitivity study by Schmidt et al. ${ }^{14}$ the inner domains of grid 1,2 and 3 contain $28^{3}, 55^{3}$ and $110^{3}$ cells, respectively. The grids and the corresponding bubble resolutions are depicted by Fig. 7 for the central plane cross section view indicated in the upper left sub-figure. The liquid fraction field is initialized such that cells entirely located within one of the bubbles are assigned a liquid fraction of $\gamma=0$ and cells entirely located in the liquid phase are assigned a value of $\gamma=1$. A sample algorithm is applied to determine the liquid fraction of those cells that are cut by any bubble interface. ${ }^{2}$ The density field is given by the linear mixture relation given by Eq. (3), where the liquid and vapor densities are assumed to be $\rho_{l}=1000 \mathrm{~kg} / \mathrm{m}^{3}$ and $\rho_{v}=0.02 \mathrm{~kg} / \mathrm{m}^{3}$, respectively. The initial pressure field is determined in exactly the same way, with the vapor pressure being $p_{v}=2340 \mathrm{~Pa}$ and the liquid pressure being equal to the far field pressure $p_{\infty}=40$ bars. This is not yet an appropriate initial condition for the liquid pressure field, which must satisfy the

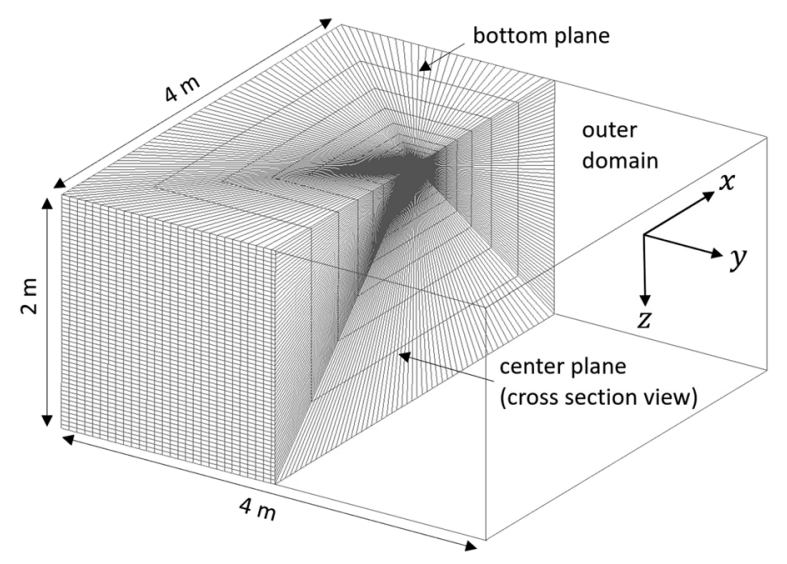

FIG. 5. Computational domain with $4 \mathrm{~m} \times 4 \mathrm{~m}$ bottom surface area and grid refinement toward the inner part of the domain.

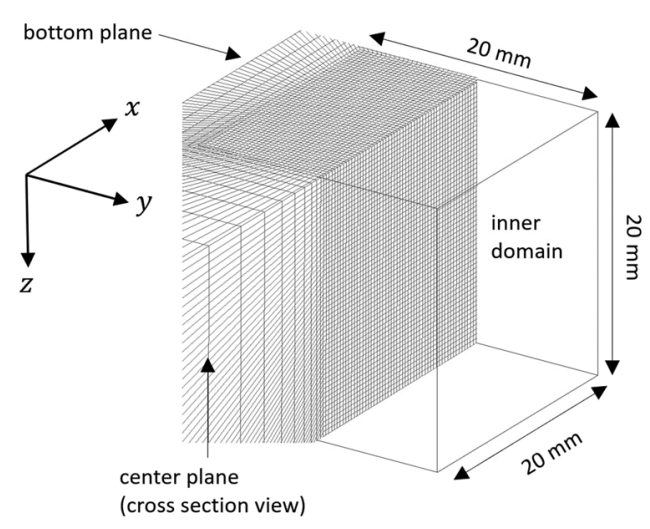

FIG. 6. Cubic inner part $(2 \mathrm{~cm} \times 2 \mathrm{~cm} \times 2 \mathrm{~cm})$ of the computational domain depicted in Fig. 4; the inner domain contains the bubble cluster depicted in Fig. 4.

Laplace equation $\nabla \cdot \nabla p=0$ in order to eliminate spurious acoustics. ${ }^{14}$ In Sec. III B, it is explained how this condition is established by the numerical flow solver during the first time step of the simulation. One close wall bubble is marked as B1 in Figs. 4 and 7, because its collapse will be shown to cause a localized high impact load on

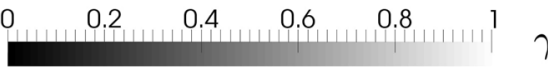

(a) bubble cluster - side view

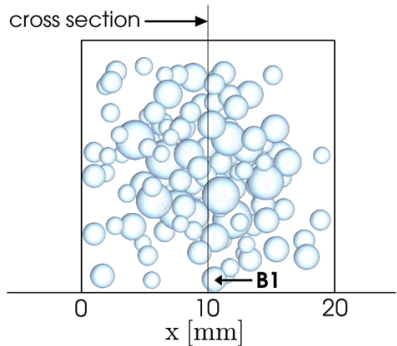

(b) Grid 1: $28^{3}$ cells in the inner domain

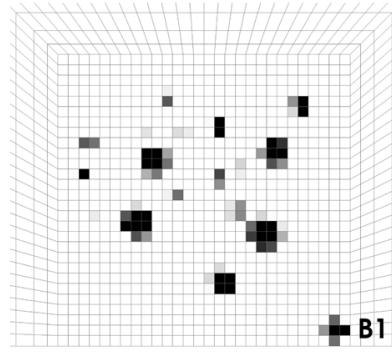

(c) Grid 2: $55^{3}$ cells in the inner domain

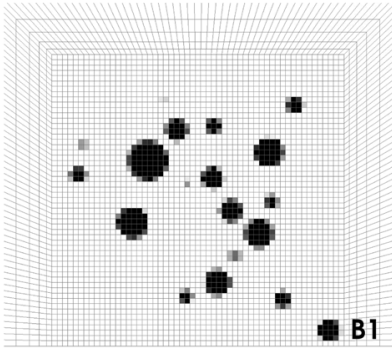

(d) Grid 3: $110^{3}$ cells in the inner domain

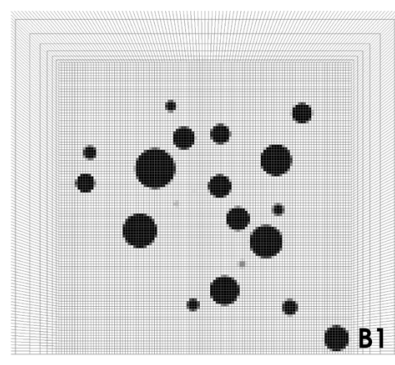

FIG. 7. Cross-sectional view (indicated in the top left) of the bubble cluster initialalized for different grid densities in the inner domain; the close wall bubble B1 is also marked in Fig. 4. 
the bottom wall which will be discussed in more detail. The driving pressure $p_{d}$ in Eq. (23) is assumed to be constant in space and time and equal to the ambient pressure $p_{\infty}$.

\section{B. Discretization schemes and iterative method}

A segregated approach involving pressure-velocity coupling ${ }^{25}$ is employed to solve the Euler equations given by Eqs. (1) and (2) for a collocated grid arrangement. The pressure-velocity coupling is achieved by solving a pressure equation, followed by a velocity correction obtained by forward substitution of the previously obtained pressure field. A beneficial feature of this method is that the pressure equation contains a Laplacian term of the pressure field, which means that the pressure equation evolves into $\nabla \cdot \nabla p=0$ at the beginning of the simulation, where the flow is still at rest. This automatically provides a consistent initial condition of the pressure field, as discussed in Sec. III A. At each time step, three inner loops are performed over the pressure-velocity coupling step. The liquid fraction transport equation given by Eq. (4) is solved separately from the pressure-velocity coupling step and placed in an outer loop, which is only performed once per time step. This configuration with only one outer loop iteration is referred to as the PISO algorithm ${ }^{25}$ in OpenFOAM. ${ }^{15}$ In order to prevent unrealistic behavior of the $\gamma$-equation due to the sharp initial pressure jump at the bubble interfaces, the original solver loop is slightly modified such that the pressure equation is solved before the liquid volume fraction transport equation, thereby providing a smooth initial condition for the $\gamma$-equation. ${ }^{10}$

The $L_{1}$ norm is employed to measure the global residual of the algebraic equations to be solved. The residual is normalized by the maximum coefficient of the corresponding matrix diagonal. ${ }^{26}$ The solution of the pressure equation and the $\gamma$-equation is considered to be sufficiently accurate if the corresponding residuals have dropped below $10^{-11}$ and $10^{-13}$, respectively. The convective term in the $\gamma$-equation and the momentum equation is discretized with the Van Leer scheme ${ }^{27}$ and the upwind-biased linear scheme, ${ }^{28}$ respectively. A linear discretization scheme as described by Jasak ${ }^{25}$ is applied to the Laplacian term in the pressure equation. Time integration is linear implicit, where the implicit treatment of the mass transfer source terms in the pressure and the $\gamma$-equation follows the procedure described by Asnaghi et al. ${ }^{2}$

The negative velocity divergence $\{\nabla \cdot \mathbf{u}\}^{-}$, needed to compute the material derivative $D \gamma / D t$ and in last instance the acoustic power radiation given by Eq. (23), is reconstructed from the face fluxes such that $(\nabla \cdot \mathbf{u}){ }_{C} V_{C}=\sum_{f} \mathbf{u}_{f} \cdot \mathbf{S}_{f}$ for each cell. The subscripts $C$ and $f$ refer to the cell center and face center location, respectively. $V_{C}$ is the cell volume, and $\mathbf{S}_{f}$ is the face area times the outward directed face normal vector. It has been shown ${ }^{2}$ that this reconstruction involves numerical errors, which would eventually violate the energy balance in the cavitation intensity approach. To at least correct for this error in an integral sense, we calculate the radiated power given by Eq. (23) based on a corrected negative velocity divergence $k\{\nabla \cdot \mathbf{u}\}_{C}^{-}$, where $k$ is determined such that the balance

$$
k \sum_{i=1}^{N_{\text {cells }}}\left[\left(\frac{D \gamma}{D t}\right)_{C, i} V_{C, i}\right]=\sum_{i=-1}^{N_{\text {cells }}}\left[\left(\frac{\partial \gamma}{\partial t}\right)_{C, i} V_{C, i}\right]
$$

is satisfied. This correction is motivated by the circumstance that the volume integral of the partial $\gamma$ time derivative, represented by the right-hand side of Eq. (32), correctly reflects the overall change of vapor volume because it directly results from solving the $\gamma$ transport equation given by Eq. (4). It is noted that $\partial \gamma / \partial t$ includes an advective component that does not contribute to the change of vapor volume. However, the advective components cancel each other out in the volume integral. By this means, the numerical errors made during the reconstruction of $\{\nabla \cdot \mathbf{u}\}^{-}$are eliminated from the integral balance and the numerical conversion of radiated power into surface impact power can be checked against the analytical prediction given by Eq. (8). Similar to the reconstruction of the cell-centered velocity divergence, the cell centered gradient of $\mathcal{E}$ in Eq. (26) is computed from the finite volume representation $(\nabla \mathcal{E})_{C} V_{C}=\sum_{f} \mathcal{E}_{f} \mathbf{s}_{f}$.

More details on how the local surface specific impact power is reconstructed from the source term distribution $\dot{e}_{\text {rad }}$ are found in previous work. ${ }^{2}$ The solution of the additional transport equation describing the spatial focusing of potential cavity energy is explicitly forwarded in time. This means that all terms on the right-hand side of Eq. (29) are assumed to be known from the previous time step $t$ such that $\partial \mathcal{E} / \partial t$ is obtained by forward substitution. The updated solution $\left.\mathcal{E}\right|_{t+\delta t}$ is then given by the first order Taylor series expansion $\left.\mathcal{E}\right|_{t+\delta t}=\left.\mathcal{E}\right|_{t}+\partial \mathcal{E} /\left.\partial t\right|_{t} \delta t+\mathcal{O}\left(\delta t^{2}\right)$. The time increment $\delta t$, by which the solution is expanded, is equal to the time step size $\Delta t$. With $\left.\dot{e}_{\text {pot }}\right|_{\nabla \cdot \mathbf{u} \leq 0}, \mathfrak{P}_{\mathbf{u}}(\nabla \mathcal{E})$ and $k$ being specified by Eqs. (23), (26), and (28), respectively, this gives

$$
\left.\mathcal{E}\right|_{t+\Delta t}=\left.\left(1-\left.\beta\right|_{t}\right)[\underbrace{\left.\dot{e}_{\text {pot }}\right|_{\nabla \cdot \mathbf{u} \leq 0} \Delta t(k-1)}_{\geq 0 \text { as } k \leq\left. 0 \wedge \dot{e}_{\mathrm{pot}}\right|_{\nabla \cdot \mathbf{u} \leq 0}<0}-\underbrace{\mathcal{E}\left(\mathfrak{P}_{\mathbf{u}}(\nabla \mathcal{E})-1\right)}_{\leq \mathcal{E} \text { as } 0 \leq \mathfrak{P}_{\mathbf{u}}(\nabla \mathcal{E}) \leq 1}]\right|_{t}
$$

for the accumulated collapse induced kinetic energy and

$$
\left.\dot{e}_{\text {rad }}\right|_{t+\Delta t}=\left.\frac{1}{\Delta t}(\beta \mathcal{E})\right|_{t}
$$

for the radiated energy. As it can be seen from Eq. (33), $\mathcal{E} \geq 0$, the updated solution for $\mathcal{E}$ is bound by 0 , which means that no negative collapse induced kinetic energy can be produced by the numerical time integration.

Furthermore, the evaluation of the terms $\{\phi(\mathcal{E})\}^{-}$and $\{\phi(\mathcal{E})\}^{+}$given by Eqs. (26) and (28), respectively, requires some special treatment because they are only to be evaluated at locations where the corresponding denominator is different from zero. To avoid a point-wise check of the denominator value, a small number $\delta$ is added to the denominator of Eq. (26) and subtracted from the denominator of Eq. (28) to prevent from division by zero. The value of $\delta$ is chosen to be $10^{-15}$ such that it does not affect the accuracy of the final result.

\section{RESULTS AND DISCUSSION}

\section{A. Sensitivity study}

In order to obtain a physically converged result for the collapse characteristic of the bubble cloud, it is essential to investigate the sensitivity of the collapse time $\tau$ with respect to the mass transfer coefficients of the cavitation model and the temporal resolution. This is done for grid 2. Figure 8 shows the evolution of total vapor volume over time for a fixed time step size $\Delta t=10^{-8} \mathrm{~s}$ and for 


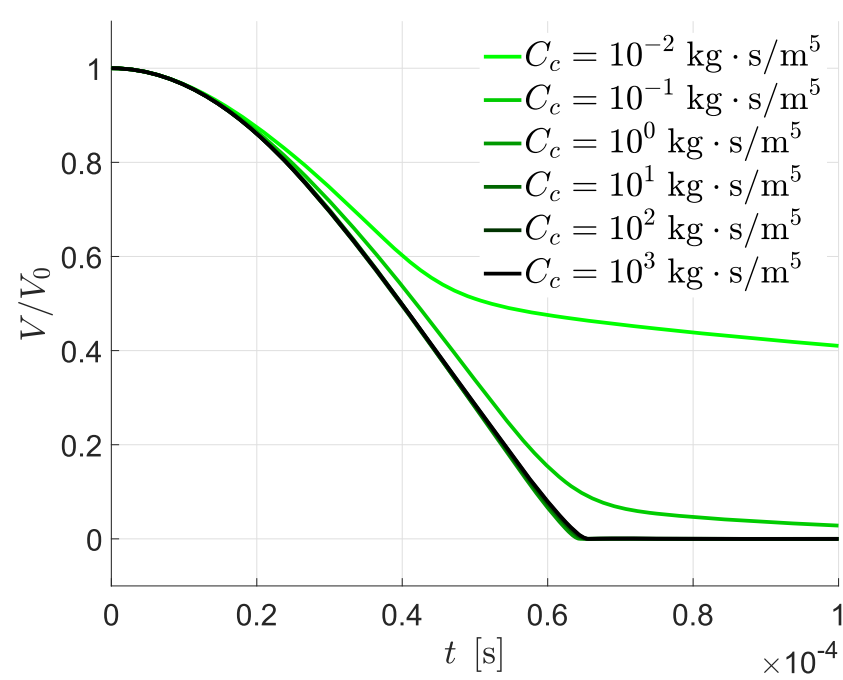

FIG. 8. Evolution of the dimensionless vapor volume over time $\left(V_{0}\right.$ is the initial vapor volume of the cloud) for grid 2 in Fig. $7, \Delta t=10^{-8}$ and a systematic variation of the condensation mass transfer constant $C_{c}$ in Eq. (4).

different magnitudes of the condensation constant $C_{c}$. For very small values of $C_{c}$, a significant delay of the collapse time is observed. In the limit $C_{c} \rightarrow 0$, no condensation can take place at all. For values of $C_{c} \geq 1 \mathrm{~kg} \mathrm{~s} / \mathrm{m}^{5}$, the curves collapse. However, this model parameter independent behavior can only be achieved if the time step $\Delta t$ is sufficiently small. Figure 9 shows the evolution of vapor volume over time for a systematic variation of $\Delta t$, where the condensation constant is kept constant at $C_{c}=1000 \mathrm{~kg} \mathrm{~s} / \mathrm{m}^{5}$. Significant delays of the collapse time are observed for large time step sizes. As $\Delta t$ approaches $10^{-8} \mathrm{~s}$, the curves converge to a time step size independent solution.

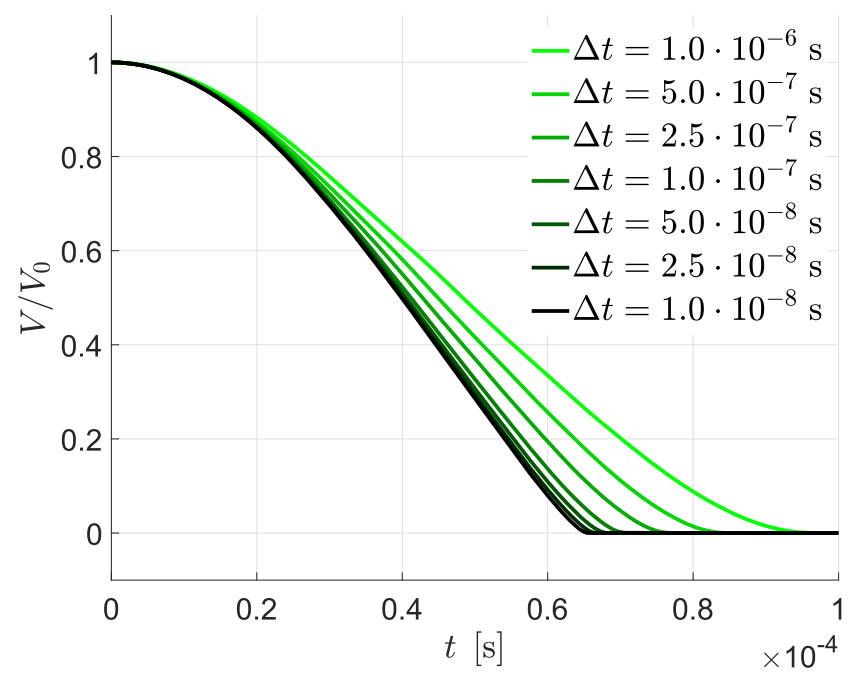

FIG. 9. Evolution of the dimensionless vapor volume over time $\left(V_{0}\right.$ is the initial vapor volume of the cloud) for grid 2 in Fig. 7, $C_{c}=1000 \mathrm{~kg} \mathrm{~s} / \mathrm{m}^{5}$ and a systematic variation of the time step size $\Delta t$.

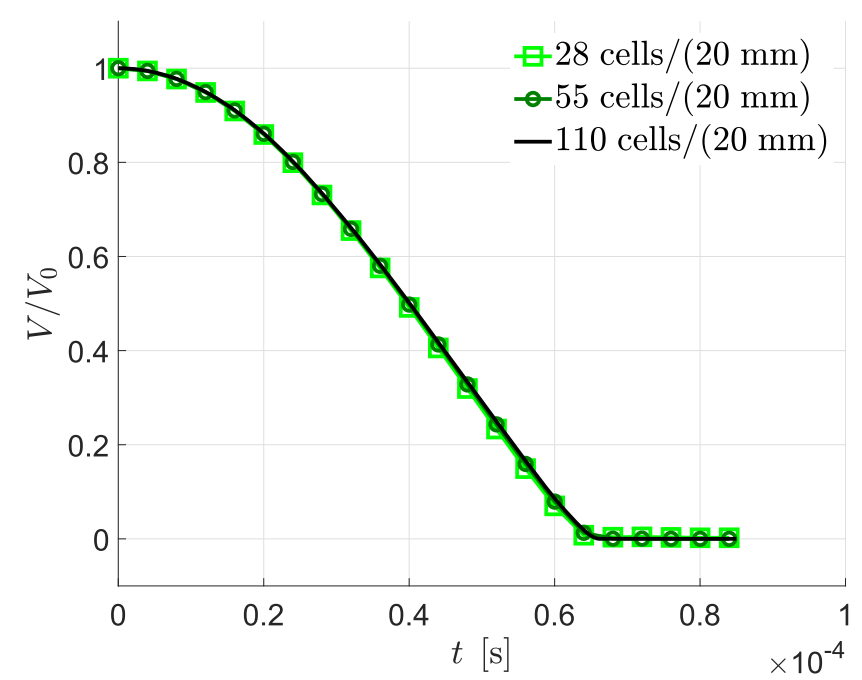

FIG. 10. Evolution of the dimensionless vapor volume over time $\left(V_{0}\right.$ is the initial vapor volume of the cloud) for $\Delta t=10^{-8}, C_{c}=1000 \mathrm{~kg} \mathrm{~s} / \mathrm{m}^{5}$ and for the three different grids depicted in Fig. 7.

Figure 10 further depicts the evolution of vapor volume over time for the three different grids in Fig. 7. It is found that the evolution of total vapor volume is practically grid insensitive for the grid densities investigated in this study. This finding is in line with the results by Schmidt et al. ${ }^{14}$ even though their results have been obtained from a fundamentally different numerical method. The results are further supported by similar findings in related studies. ${ }^{30-32}$ The collapse time of the bubble cloud is identified by a change of sign of $\dot{V}$ at the final collapse stage and found to be $\tau=6.5 \times 10^{-5} \mathrm{~s}$, which is in good agreement with the results by Schmidt et al. ${ }^{14}$ The insensitivity of $\tau$ with respect to the grid density is explained by the circumstance that the bubble population density of the cloud is so high that is does not allow for any significant pressure recovery between the bubbles. This is illustrated by Fig. 11, depicting cross sectional views of the pressure field at $t=0.08 \tau$. For inertia driven cavitating flows, the finding from this sensitivity analysis can be generalized in so far that the time step size must be chosen as small as necessary and the mass transfer coefficients as large as necessary. If this condition is met, the mass transfer model always has enough capacity to achieve phase transition within the time scale that is governed by inertia driven flow and the local phase transition event is as far resolved in time that no truncation errors occur. Based on the sensitivity study, the configuration of $C_{c}=10 \mathrm{~kg} \mathrm{~s} / \mathrm{m}^{5}, \Delta t=10^{-8} \mathrm{~s}$, and grid 2 is found to provide a physically converged solution.

\section{B. Physical analysis}

Figure 12 depicts the evolution of the dimensionless accumulated surface energy $E_{S} / E_{\text {pot }, 0}$, obtained from integration of the surface specific impact power over time and over the entire $4 \mathrm{~m} \times 4 \mathrm{~m}$ bottom surface area. The circular markers represent the curve obtained from the original cavitation intensity approach, hence, without applying the energy focusing equation introduced in Sec. II D. The other curves represent the results obtained from 


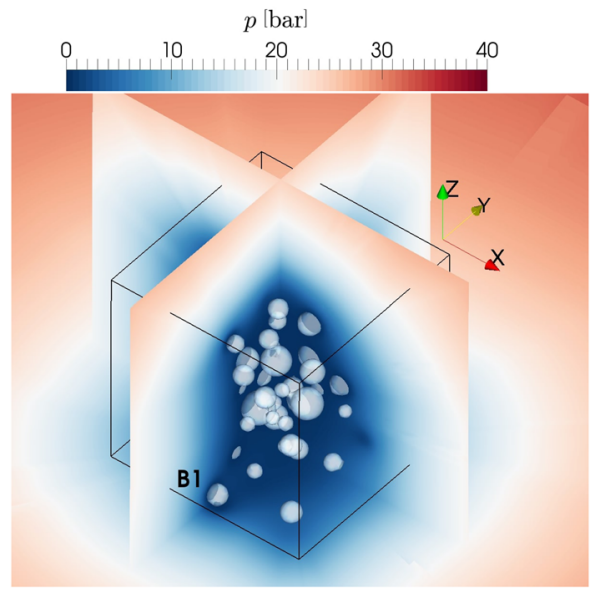

FIG. 11. $y=0.5$ isosurfaces of the vapor bubbles at time instant $t=0.08 \tau$ and cross sectional views on the corresponding pressure field for grid 2 in Fig. 7 , $C_{c}=1000 \mathrm{~kg} \mathrm{~s} / \mathrm{m}^{5}$ and $\Delta t=10^{-8}$

the potential energy focusing approach for different values of the focusing parameter $h$ in Eq. (30). If no potential energy focusing is applied, the percentage amount of $E_{S}$ relative to $0.5 E_{\mathrm{pot}, 0}$ corresponds exactly to the percentage of dimensionless volume $V / V_{0}$ by which the cavity has shrunk. When the potential energy focusing is applied, the temporal evolution of the surface impact is governed by the focusing parameter $h$, where the impact is more and more delayed toward the final collapse stage as $h$ increases. In all cases, however, the amount of accumulated surface energy converges to the analytically predicted value of $50 \%$ initial potential energy [see Eq. (8)] with reasonable accuracy. In the case of

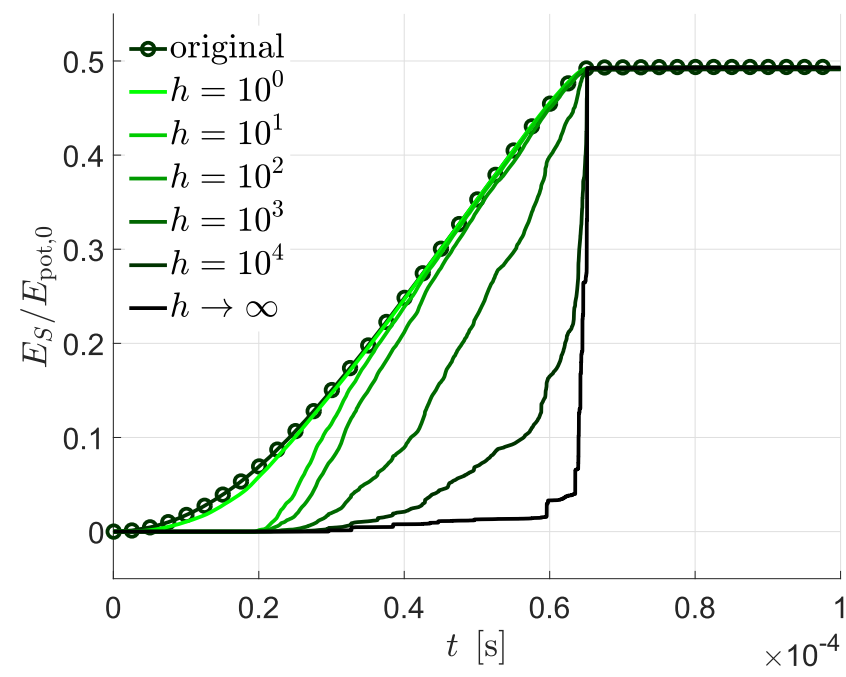

FIG. 12. Evolution of the dimensionless energy accumulated on the entire bottom surface; the "original" curve represents the distribution without focusing and the remaining curves the distributions with focusing of the potential energy for a systematic variation of the focusing parameter $h$ in Eq. (30). $h \rightarrow \infty$, which is computed from Eq. (31), the major amount of energy is focused toward the final collapse stage and then released in a rather sudden step. The lowest focusing parameter value $h=1$ hardly appears to have any effect on the evolution of the accumulated surface energy at all because it can only slightly delay the release of potential cavity energy. For the parameter range $10 \leq$ $h \leq 100$, the energy release is effectively delayed up to the time instant where the first isolated bubbles in the outer periphery of the cloud have collapsed. From this moment on, the curves approach the original curve again. This behavior is explained by the focusing parameter $h$ being large enough to focus the potential energy within the isolated bubbles but being too small to effectively transport the potential energy across the liquid phase. With further increasing values of $h$, the energy release in the liquid phase is effectively suppressed so that the corresponding curves approach the $h \rightarrow \infty$ case.

In view of the acoustic pressure analysis, it is noted that the total pressure computed by Schmidt et al. ${ }^{14}$ depicted by the red solid line in Fig. 14, is not entirely comparable with the acoustic pressure $p_{a}$. The most obvious difference is that the total pressure must be equal to the far field pressure $p_{\infty}$ once the pressure perturbations caused by the cloud collapse have decayed, whereas the acoustic pressure is a pressure perturbation itself which tends to zero after the collapse event. At the beginning of the collapse, the total wall pressure in close vicinity of the vapor bubble cloud is significantly lower than the far field pressure. This is explained by the fact that the liquid phase must satisfy the Laplace equation of pressure initially, where the close wall distance of the vapor bubble cloud hardly allows the liquid pressure to recover on the bottom wall. The collapse peak pressure computed by Schmidt et al., ${ }^{14}$ however, is supposed to be governed by the energy transported by the shock wave through the nearly incompressible liquid phase and should, therefore, exhibit a similar characteristic as the acoustic peak pressure reconstructed from the cavitation intensity approach.

Figure 13 shows the acoustic pressure signals obtained from averaging the acoustic pressure $p_{a}$ over the numerical pressure sensor depicted in Fig. 4. The subfigure entitled "original" represents the signal obtained from the original approach without energy focusing. Compared to the results by Schmidt et al., ${ }^{14}$ a strong smearing of the signal is observed. The pronounced peak at the end of the collapse that is observed in the results by Schmidt et al. ${ }^{14}$ is not present in the "original" acoustic pressure signal at all. This clearly demonstrates the inability of the original cavitation intensity approach to reflect the instantaneous energy balance, as discussed in Sec. II D. Figure 13 further depicts the acoustic pressure signal evaluated on the numerical pressure sensor for a systematic variation of the focusing parameter $h$. As a result of the delayed power radiation with increasing $h$, a peak event forms at the end of the cloud collapse. The peak intensifies with increasing $h$. For very large $h$, various spurious high amplitude peaks are observed before the final collapse stage. They are identified as spurious peaks because the fact that the by far largest energy portion is impacting the surface at the end of the collapse event (see Fig. 12) implies that there is no significant energy content in the peaks before the final collapse stage. This is further supported by Fig. 14, showing a 200th order Fourier approximation of the $p_{a}$-signal for $h \rightarrow \infty$. Even for this high order approximation, the spurious peaks before the final collapse stage are filtered 

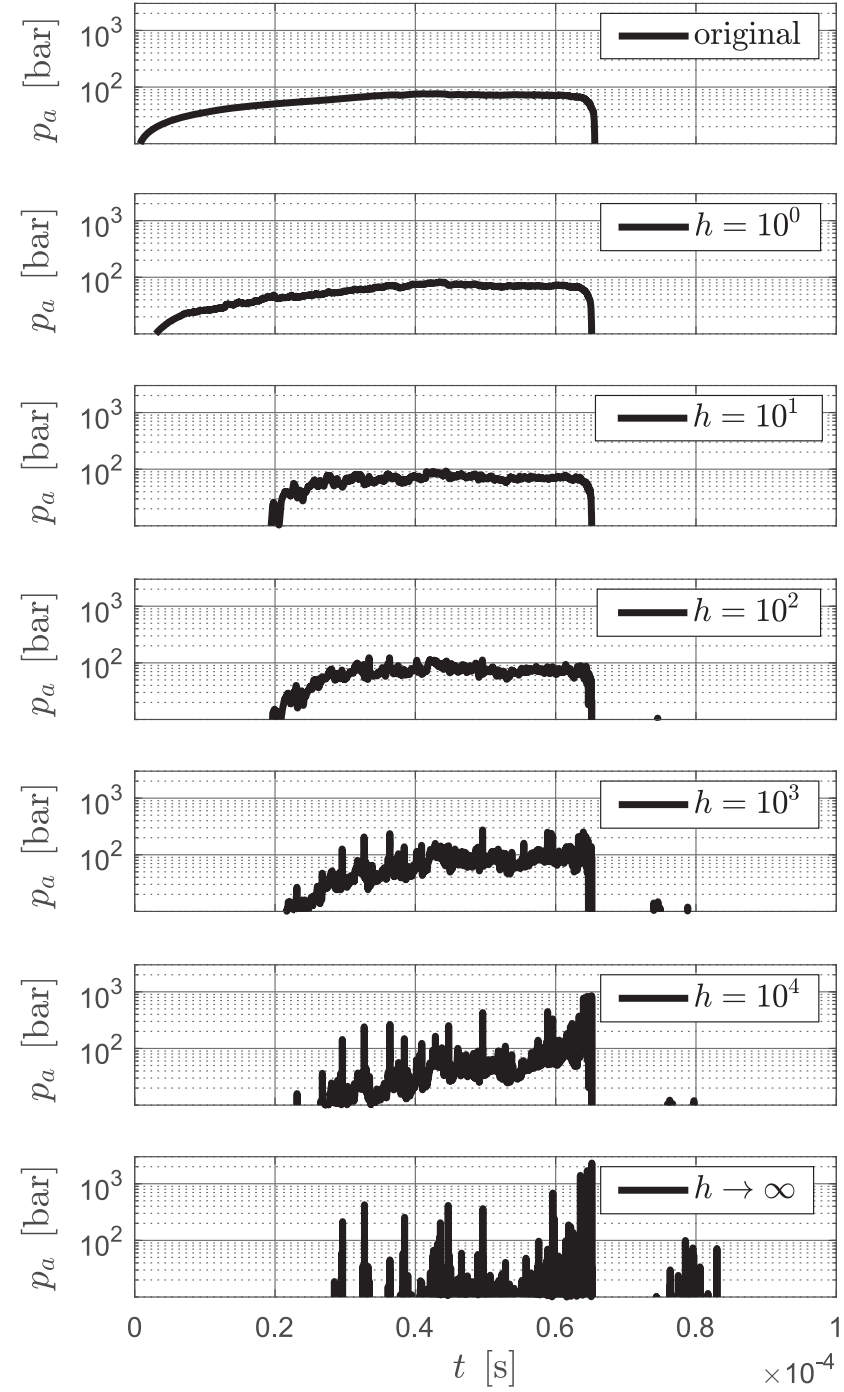

FIG. 13. Evolution of the acoustic pressure signal averaged over the numerical pressure sensor surface depicted in Fig. 4 for a systematic variation of the energy focusing parameter $h$ in Eq. (30); the "original" curve represents the distribution without potential energy focusing.

out. Figure 14 further depicts the total pressure signal computed by Schmidt et al. ${ }^{14}$ for reference. It is observed that the total pressure peak value occurs somewhat later than the acoustic pressure peak. This delay is due to the time that it takes for the pressure wave to propagate from the cloud collapse center to the bottom wall in the compressible simulation by Schmidt et al. ${ }^{14}$ where the wave propagation speed of the associated wave in the cavitation intensity model is assumed to be infinite. A rough estimation of the wave propagation time can be made by assuming a propagation speed of $c_{l}=1500 \mathrm{~m} / \mathrm{s}$ and by assuming the cloud collapse center to be in the middle of the inner domain, which gives an approximate propagation time of $6.7 \times 10^{-6} \mathrm{~s}$. The impact time of the acoustic pressure peak is also found to be significantly smaller than the impact time of

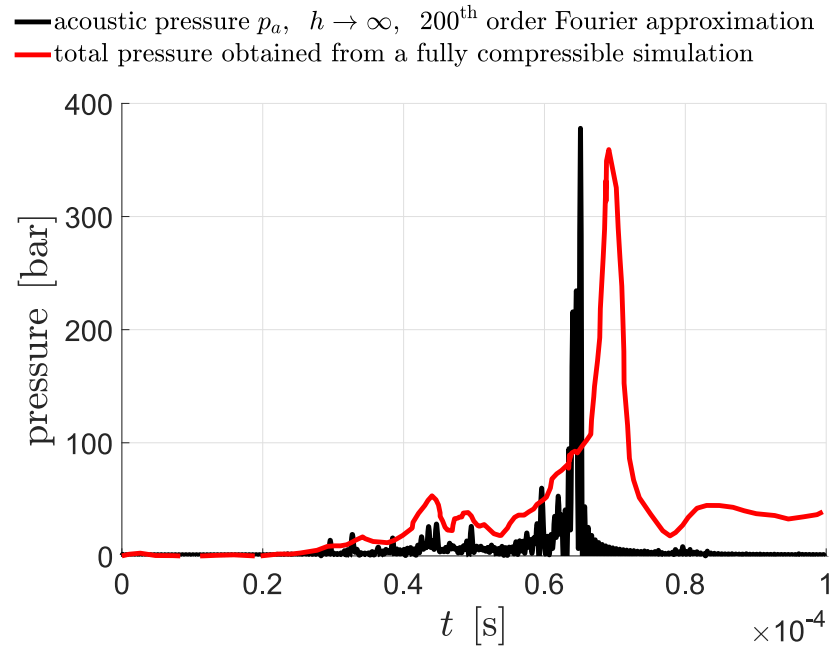

FIG. 14. Comparison of the acoustic pressure signal obtained from the cavitation intensity model for maximum energy focusing $(h \rightarrow \infty)$ with the total pressure signal computed by Schmidt et al.

the total pressure peak by Schmidt et al. ${ }^{14}$ As discussed in Sec. II D, this difference is attributed to the tendency of the energy focusing equation to release the accumulated potential energy in a sudden step at the final collapse stage as $h \rightarrow \infty$.

A measure of the impact aggressiveness is given by the distribution of surface specific energy accumulated throughout the cloud collapse. Figure 15 depicts the accumulated surface energy distribution on the bottom wall underneath the cloud, normalized by the cloud collapse time $\tau=6.5 \times 10^{-5}$ s. The top figure shows the distribution obtained from the original cavitation intensity model without potential energy focusing and the lower figure the distribution obtained from applying the focusing approach described in Sec. II D for $h \rightarrow \infty$ (maximum focusing). In both cases, one distinct isolated footprint is observed, which is caused by the isolated close wall bubble marked as B1 in Fig. 4. The energy focusing approach predicts only one more isolated footprint, which is located outside the numerical pressure sensor surface and which has been caused by a bubble initially located in the outer periphery of the cloud. The original approach predicts another isolated footprint located on the numerical pressure sensor surface and generally tends to leave a more asymmetric impact pattern. With the major part of the initial potential cloud energy being focused toward the collapse center, the focusing approach leaves a rather axisymmetric footprint on the numerical pressure sensor as would be observed if the impact was caused by an isolated point source. The surface energy distribution obtained from the energy focusing approach is also of larger magnitude as compared to the original approach. Another effect of the collective focusing of potential energy is that the peak value of the accumulated surface energy distribution is caused by the collective collapse event, whereas the original approach predicts the peak value in the vicinity of the isolated collapse of bubble B1.

The potential energy focusing is further illustrated by Fig. 16, depicting the distribution of accumulated volume specific kinetic 

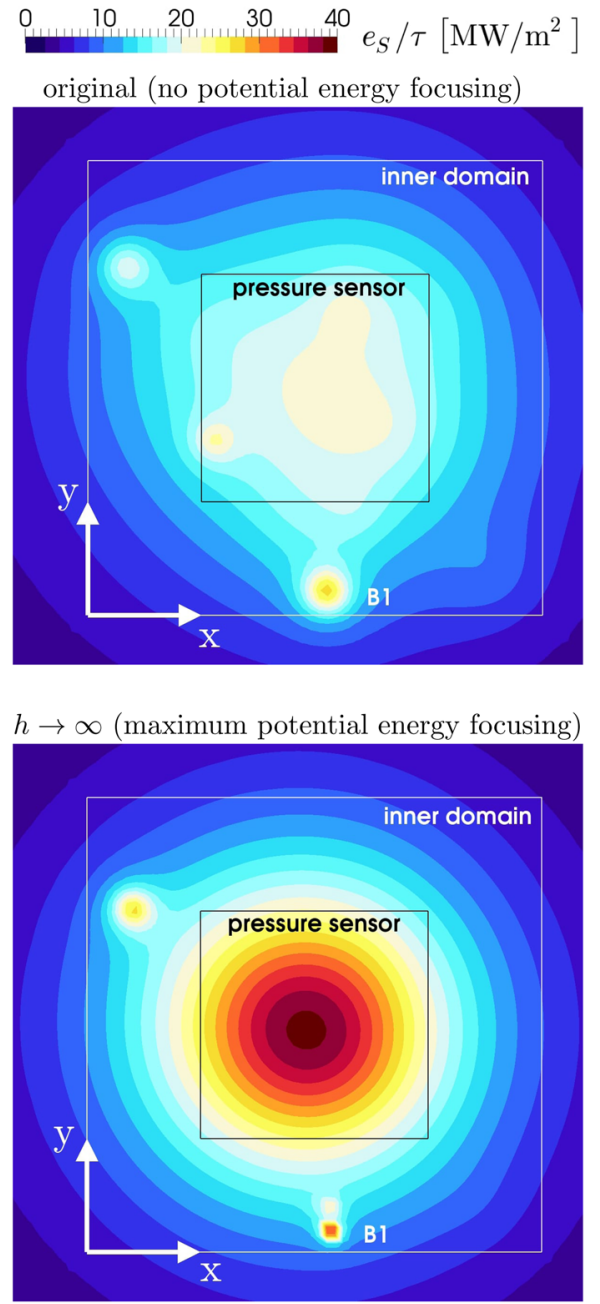

FIG. 15. Distribution of the accumulated surface specific energy normalized by the cloud collapse time $\tau=6.5 \times 10^{-5} \mathrm{~s}$ for the original model without potential energy focusing (top) and maximum energy focusing $h \rightarrow \infty$ (bottom); the accumulated surface energy is obtained from time integration of the surface specific impact power.

energy $\mathcal{E}$ obtained from Eq. (33). The bubble interfaces are indicated by the red solid line. It can be seen how the collapse induced kinetic energy accumulation starts in the outer cloud periphery and how it focuses and intensifies toward the cloud collapse center as the collapse time $\tau$ is approached. One isolated region of pronounced kinetic energy accumulation prior to the final collapse stage is observed at the collapse location of bubble B1 in Fig. 4. In agreement with the results by Schmidt et al., ${ }^{14}$ the bubble cloud is found to rather collapse like a structure of homogeneous water-vapor mixture in the sense that the bubbles consecutively collapse from the outer peripheries to the inner ones. Indeed, it has been shown by Schmidt et al. ${ }^{14}$ that, in this particular case, the bubble cloud can be replaced by a homogeneous structure of equivalent volume fraction without changing the collapse characteristic. As shown by

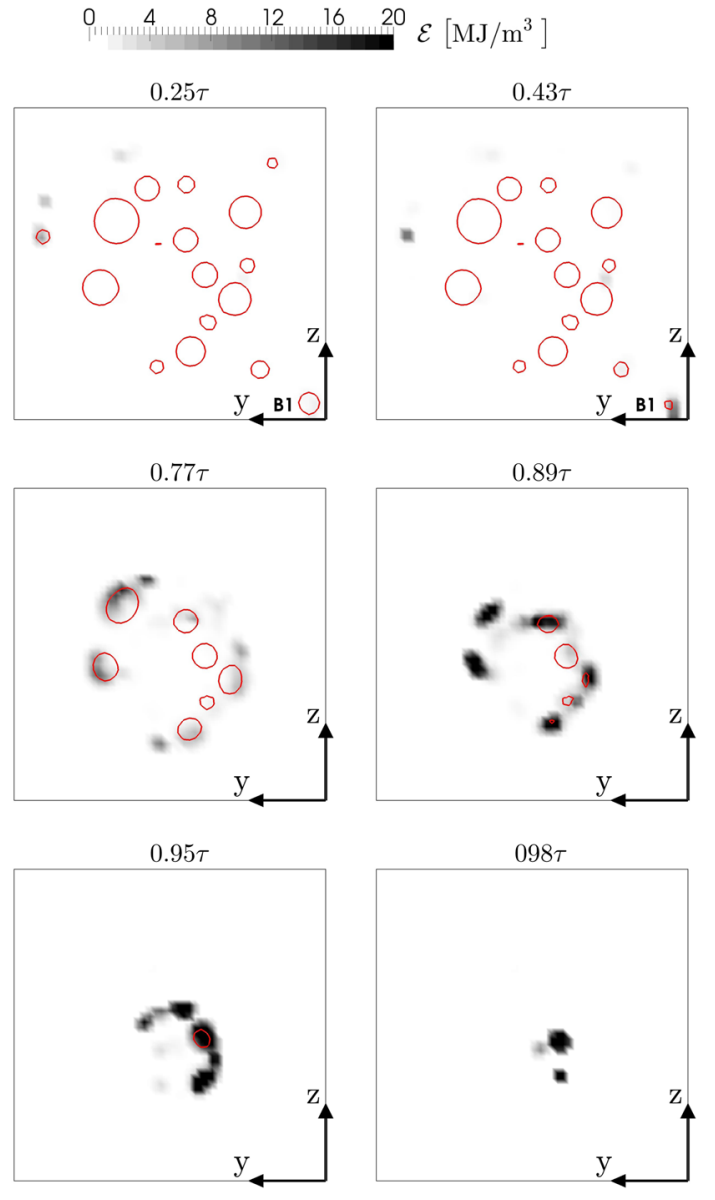

FIG. 16. Cross-sectional view (as indicated in Fig. 7) of the distribution of the accumulated volume specific energy $\mathcal{E}$ [see Eq. (33)] at different time instants relative to the cloud collapse time $\tau=6.5 \times 10^{-5}$; the black outline represents the cubic inner domain $(2 \mathrm{~cm} \times 2 \mathrm{~cm} \times 2 \mathrm{~cm})$ and the red solid line the $\gamma=0.5$ isosurface of the instantaneous bubble cloud.

Wang and Brennen, ${ }^{12}$ it strongly depends on the density of the bubble population whether a bubble cloud exhibits this collective behavior. In sparsely populated clouds, the bubbles tend to behave as individual units. ${ }^{12}$ Based on their early numerical computations on collapsing vapor bubble clouds, Wang and Brennen ${ }^{12}$ propose that the focusing of potential energy across the individual bubbles into the inner peripheries of a densely populated cloud is governed by an inward directed bubbly shock or condensation shock wave. As discussed in Sec. II D, this mechanism is reflected by Eq. (30), which suppresses the source radiation until the pressure in the liquid phase has exceeded the driving pressure. Thus, the potential cavity energy is accumulated and transported on the low pressure side of the bubbly shock front. This also means that the capability of the improved cavitation intensity model to reflect the potential energy focusing driven by the inward directed condensation shock strongly depends on the capability of the flow solver to resolve this flow phenomenon. It has been shown that even 
semiempirical mass transfer approaches involving adjustable model coefficients can resolve condensation shock states ${ }^{10,31}$ if the prerequisites discussed in Sec. IV A are met. Indeed, the formation of an inward directed bubbly shock as described by Wang and Brennen ${ }^{12}$ is indicated by Fig. 17, depicting a cross-sectional view of the distribution of the total pressure (left) and the velocity magnitude (right) for different time instants. It can be seen how a discontinuity in both the pressure and the velocity field forms across the outer cloud periphery at $t=0.83 \tau$, which gets more pronounced as $\tau$ is approached.

Despite the fact that the impact load characteristics are well captured by the method presented in this study, some uncertainties remain. One uncertainty concerns the driving pressure $p_{d}$ in Eq. (23), which is assumed to be constant and equal to the ambient pressure $p_{\infty}$ for the entire cloud surface. However, due to the effects of wall interaction and the interaction of rather isolated bubbles with the close by bubble cloud, the driving pressure distribution is in fact neither constant in space nor constant in time and the

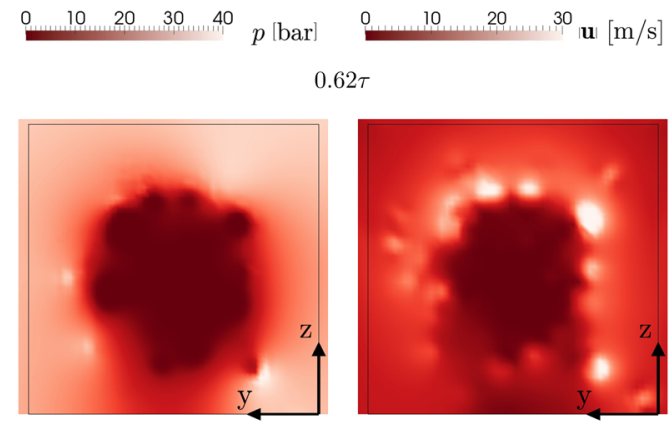

$0.83 \tau$

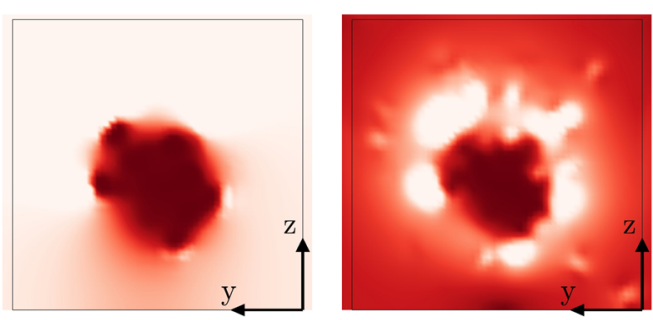

$0.92 \tau$

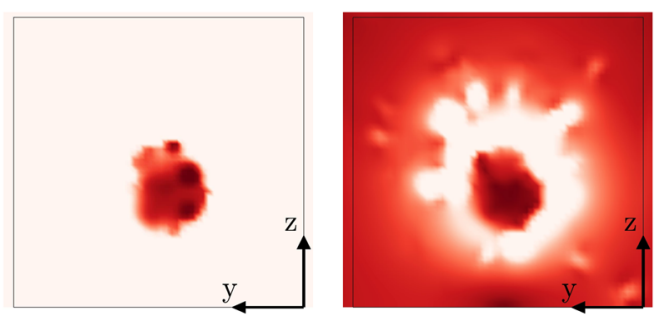

FIG. 17. Cross-sectional view (as indicated in Fig. 7) of the distribution of the total pressure (left) and the velocity magnitude for different time instants relative to the cloud collapse time $\tau=6.5 \times 10^{-5}$; the black outline represents the cubic inner domain $(2 \mathrm{~cm} \times 2 \mathrm{~cm} \times 2 \mathrm{~cm})$. assumption that $p_{d}\left(t, \mathbf{x}_{P}\right)=p_{\infty}$ can only be an approximation of the actual condition experienced by the collapsing cloud. The problem of not exactly knowing the effective driving pressure $p_{d}$ in the cavitation intensity approach has been discussed in detail in previous work. ${ }^{2}$ It is also noted that as discussed in Sec. II D, one can still not expect an entirely time accurate representation of the acoustic impact signal. The delay of the collapse impact toward the final collapse stage due to the spatial focusing of potential cavity energy reflects the bubble cloud dynamics more accurately than the original cavitation intensity approach, but the exact events at the final collapse stage are beyond the model resolution. As a result, the model theoretically allows us to transfer the shock wave energy at an infinitely small impact duration. It is also mentioned that the transmitted shock wave energy computed in the present study is associated with the idealized situation of zero noncondensable gas content. The relation between gas content and shock wave energy derived by Tinguely et al., ${ }^{11}$ Fortes-Patella et al., ${ }^{33}$ and Brennen ${ }^{34}$ might be employed in future work to complement the model by the effect of noncondensable gas. Finally, the reconstruction of the velocity divergence needed for the computation of the local acoustic power source terms is subjected to numerical errors, which is why the correction given by Eq. (32) has been applied. By means of this correction, the volume integrated energy balance could be satisfied in the present study. As already discussed in previous work, ${ }^{2}$ however, this correction cannot be applied to any arbitrary flow situation. Therefore, a best possible reconstruction of the velocity divergence field is a key factor for the reliability of the method presented in this study.

\section{CONCLUSION}

A novel methodology is presented in this study, which allows for the computation of the acoustic power and pressure from the change of potential energy of collapsing vapor cavities in a liquid. The essential feature that has been added to the original model formulation found in previous works ${ }^{1,2}$ is an energy focusing equation which can realistically focus the potential cavity energy into the collapse center prior to its conversion into acoustic power. Compared to the previous approaches, the physics of a cavity collapse are captured more accurately by the new model because it accounts for the fact that the potential cavity energy is mostly converted into kinetic energy of the surrounding liquid during the collapse and that the conversion into acoustic power only takes place at the final collapse stage. $^{11,13}$

The capability of the new model is demonstrated for the collapse of a vapor bubble cloud for which a more realistic and time accurate acoustic pressure signal is obtained. From the analysis of the acoustic pressure signal and the accumulated surface energy distribution, it has been shown that the spatial focusing of cavity energy caused by the collective cloud collapse ${ }^{12}$ is all in all well captured. An energy focusing parameter $h$ has been introduced to demonstrate the gradual transition of the model from the previous nonfocusing formulation $(h=0)$ to the maximal energy focusing form as $h \rightarrow \infty$. The latter case fully adopts the instantaneous energy balance during the cavity collapse, describing how the reduction of potential cavity energy feeds into kinetic energy of the surrounding liquid prior to the final collapse stage. ${ }^{13}$ Therefore, the limit $h \rightarrow \infty$, where acoustic energy radiation only takes place at the final cavity collapse stage, is 
considered as the physically correct model configuration for future studies.

Some uncertainties concerning the potential energy content remain because the distribution of the collapse driving pressure is not exactly known. Nevertheless, the methodology presented in this study can be relevant for the prediction of cavitation erosion risk in engineering applications because the control of the energy balance allows for a reliable and physical prediction of the surface impact distribution as long as the dynamics of the larger scale cavitating structures are sufficiently well captured by the computational grid.

\section{SUPPLEMENTARY MATERIAL}

See supplementary material for data files to reproduce the $h \rightarrow \infty$ surface energy curve in Fig. 12, the $h \rightarrow \infty$ acoustic pressure signal in Fig. 13, its 200th order Fourier approximation in Fig. 14, and the $h \rightarrow \infty$ surface energy distribution depicted in Fig. 15. The material further includes a data file with the bubble positions and corresponding radii to reproduce the initial bubble cloud depicted in Fig. 4. This data set has kindly been provided by TUM/AER (see Schmidt et al. ${ }^{14}$ ) in private communication as part of the EU H2020 CaFE Project (Grant Agreement No. 642536). Please refer to the work of Schmidt et al. ${ }^{14}$ when using the bubble cloud specifications.

\section{ACKNOWLEDGMENTS}

This research is funded by the European Union Horizon 2020 Research and Innovation programme, Grant Agreement No. 642536, as well as by the MARIN (Maritime Research Institute Netherlands) Academy. We would also like to thank Dr. Steffen Schmidt [Technical University of Munich, Institute of Aerodynamics and Fluid Mechanics (TUM/AER)] for sharing the bubble cloud data that has been used in this research. Furthermore, we highly appreciate the inspiring discussions on bubble cloud dynamics together with Dr. Steffen Schmidt and Ebrahim Ghahramani (Chalmers University of Technology, Department of Mechanics and Maritime Sciences) as part of the EU H2020 CaFE Project.

\section{REFERENCES}

${ }^{1}$ C. Leclercq, A. Archer, R. F. Patella, and F. Cerru, "Numerical cavitation intensity on a hydrofoil for 3D homogeneous unsteady viscous flows," Int. J. Fluid Mach. Syst. 10, 254-263 (2017).

${ }^{2}$ S. Schenke and T. van Terwisga, "An energy conservative method to predict the erosive aggressiveness of collapsing cavitating structures and cavitating flows from numerical simulations," Int. J. Multiphase Flow 111, 200-218 (2019).

${ }^{3}$ F. G. Hammitt, "Observations on cavitation damage in a flowing system," J. Basic Eng. 85, 347-356 (1963).

${ }^{4}$ A. Vogel and W. Lauterborn, "Acoustic transient generation by laser-produced cavitation bubbles near solid boundaries,” J. Acoust. Soc. Am. 84, 719-731 (1988).

${ }^{5}$ F. Pereira, F. Avellan, and P. Dupont, "Prediction of cavitation erosion: An energy approach," J. Fluids Eng. 120, 719-727 (1998).

${ }^{6}$ R. F. Patella and J.-L. Reboud, "A new approach to evaluate the cavitation erosion power,” J. Fluids Eng. 120, 335-344 (1998).

${ }^{7}$ R. F. Patella, J.-L. Reboud, and L. Briançon-Marjollet, "A phenomenological and numerical model for scaling the flow agressiveness in cavitation erosion," in Cavitation Erosion EROCAV Workshop (Val de Reuil, France, 2004).
${ }^{8}$ R. F. Patella, A. Archer, and C. Flageul, "Numerical and experimental investigations on cavitation erosion," IOP Conf. Ser.: Mater. Sci. Eng. 15, 022013 (2012).

${ }^{9}$ M. H. Arabnejad and R. Bensow, "A methodology to identify erosive collapse events in the incompressible simulation of cavitating flows," in Proceedings of the 20th Numerical Towing Tank Symposium (Curran Associates, Wageningen, The Netherlands, 2017).

${ }^{10} \mathrm{~S}$. Schenke and T. J. C. van Terwisga, "Erosive aggressiveness of collapsing cavitating structures," in Proceedings of the 10th International Symposium on Cavitation (The American Society of Mechanical Engineers (ASME), Baltimore, Maryland, USA, 2018).

${ }^{11}$ M. Tinguely, D. Obreschkow, P. Kobel, N. Dorsaz, A. de Bosset, and M. Farhat, "Energy partition at the collapse of spherical cavitation bubbles," Phys. Rev. E 86, 046315 (2012).

${ }^{12}$ Y.-C. Wang and C. Brennen, "Numerical computation of shock waves in a spherical cloud of cavitation bubbles," J. Fluids Eng. 121, 872-880 (1999).

${ }^{13}$ D. Obreschkow, P. Kobel, N. Dorsaz, A. de Bosset, C. Nicollier, and M. Farhat, "Cavitation bubble dynamics inside liquid drops in microgravity," Phys. Rev. Lett. 97, 094502 (2006).

${ }^{14}$ S. J. Schmidt, M. Mihatsch, M. Thalhamer, and N. A. Adams, in Advanced Experimental and Numerical Techniques for Cavitation Erosion Prediction, edited by G. Chahine, K.-H. Kim, J.-P. Franc, and A. Karimi (Springer, 2014), Vol. 106, Chap. 10, pp. 329-343.

${ }^{15}$ OpenFOAM, Openfoam website, 2019.

${ }^{16}$ S. Schenke and T. J. C. van Terwisga, "Simulating compressibility in cavitating flows with an incompressible mass transfer flow solver," in Proceedings of the 5th International Symposium on Marine Propulsors (VTT Technical Research Center of Finland Ltd., Espoo, Finland, 2017).

${ }^{17}$ C. L. Merkle, J. Z. Feng, and P. E. O. Buelow, "Computational modeling of the dynamics of sheet cavitation," in Proceedings of the 3rd International Symposium on Cavitation (Grenoble, France, 1998).

${ }^{18}$ J. P. Franc and J. M. Michel, Fundamentals of Cavitation, Fluid Mechanics and its Applications (Springer Netherlands, 2006).

${ }^{19}$ Lord Rayleigh, "VIII. On the pressure developed in a liquid during the collapse of a spherical cavity," London Edinburgh Dublin Philos. Mag. J. Sci. 34, 94-98 (1917).

${ }^{20}$ R. H. Cole, Underwater Explosions (Princeton University Press, Princeton, 1948).

${ }^{21}$ M. S. Plesset and R. B. Chapman, "Collapse of an initially spherical vapour cavity in the neighbourhood of a solid boundary," J. Fluid Mech. 47, 283-290 (1971).

${ }^{22}$ C. Flageul, R. F. Patella, and A. Archer, "Cavitation erosion prediction by numerical cavitation," in Proceedings of the 14th International Symposium on Transport Phenomena and Dynamics of Rotating Machinery (Honolulu, HI, USA, 2012).

${ }^{23}$ M. A. Maiga, O. Coutier-Delgosha, and D. Buisine, "A new cavitation model based on bubble-bubble interactions," Phys. Fluids 30, 123301 (2018).

${ }^{24}$ M. S. Mihatsch, S. J. Schmidt, and N. A. Adams, "Cavitation erosion prediction based on analysis of flow dynamics and impact load spectra," Phys. Fluids 27, 103302 (2015).

${ }^{25} \mathrm{H}$. Jasak, Error analysis and estimation for the finite volume method with applications to fluid flows, Ph.D. thesis, Imperial College, 1996.

${ }^{26}$ F. Moukalled, L. Mangani, and M. Darwish, The Finite Volume Method in Computational Fluid Dynamics: An Advanced Introduction with OpenFOAM and Matlab, 1st ed. (Springer Publishing Company, Incorporated, 2015).

${ }^{27}$ B. van Leer, "Towards the ultimate conservative difference scheme. II. Monotonicity and conservation combined in a second-order scheme," J. Comput. Phys. 14, 361-370 (1974).

${ }^{28}$ R. F. Warming and R. M. Beam, "Upwind second-order difference schemes and applications in unsteady aerodynamic flows," in Proceedings of the 2nd Computational Fluid Dynamics Conference (Hartford, Connecticut, USA, 1975). 
${ }^{29}$ A. Asnaghi, E. Jahanbakhsh, and M. S. Seif, "Unsteady multiphase modeling of cavitation around NACA 0015," J. Marine Sci. Tech. 18, 689-696 (2010).

${ }^{30} \mathrm{M}$. Bhatt, A. Gnanaskandan, and K. Mahesh, "Evaluation of finite rate homogenous mixture model in cavitation bubble collapse," J. Phys.: Conf. Ser. 656, 012136 (2015).

${ }^{31}$ P. Koukouvinis and M. Gavaises, "Simulation of throttle flow with two phase and single phase homogenous equilibrium model," J. Phys.: Conf. Ser. 656, 012086 (2015).
${ }^{32}$ E. Ghahramani, M. H. Arabnejad, and R. E. Bensow, "A comparative study between numerical methods in simulation of cavitating bubbles," Int. J. Multiphase Flow 111, 339-359 (2019).

${ }^{33}$ R. Fortes-Patella, G. Challier, J.-L. Reboud, and A. Archer, "Energy balance in cavitation erosion: From bubble collapse to indentation of material surface," Journal of Fluids Engineering 135, 011303 (2013).

${ }^{34} \mathrm{C}$. Brennen, "The dynamic balances of dissolved air and heat in natural cavity flows," J. Fluid Mech. 37, 115-127 (1969). 